\title{
Synthesis and self-assembly studies of a new AIEE probe and its application in sensing amyloid fibrillation
}

Nidhi Gour, Vivek Shinh Kshtriya, Shradhey Gupta, Ramesh, Singh, Dhawal Patel, khashti Ballabh Joshi Submitted date: 21/06/2019 - Posted date: 21/06/2019

Licence: CC BY-NC-ND 4.0

Citation information: Gour, Nidhi; Kshtriya, Vivek Shinh; Gupta, Shradhey; Singh, Ramesh,; Patel, Dhawal; Joshi, khashti Ballabh (2018): Synthesis and self-assembly studies of a new AIEE probe and its application in sensing amyloid fibrillation. ChemRxiv. Preprint.

We report self-assembly and photophysical properties of a new pyridothiazole based aggregation-induced-emission enhancement (AIEE) luminogen

4-(5-methoxy-thiazolo[4,5-b]pyridin-2-yl)benzoic acid (PTC1) and its application for the sensitive detection and monitoring of amyloid fibrillation. The self-assembling properties of the new AIEE probe are extensively studied by AFM and it was noted that as aggregation increases there is enhancement of fluorescence. The fluorescence of PTC1 is quenched upon addition of cupric $\left(\mathrm{Cu}^{2+}\right)$ ions while the fluorescence is regenerated in the presence of amyloid fibers. AFM studies reveal that PTC1 self associate/aggregate to hairy micelle structures which gets disrupted on the addition of $\mathrm{Cu}^{2+}$ and again reassembles in the presence of amyloid fibers. Hence, the fluorescence quenching and regeneration may be attributed to disaggregation and AIE respectively. Further, a comparative analysis of the performance of PTC1 is done with the conventional ThT which confirms it to be a more sensitive probe for the detection of amyloid both in the presence and absence of $\mathrm{Cu}^{2+}$. Of note, a very simple, facile and cost-effective methodology for the detection of amyloid fibres is presented, wherein fluorescence quenching/enhancement can be visualized under UV without the use of sophisticated instrumentation techniques. To the best of our knowledge and literature survey, this is first report wherein the self-assembling properties of AIEE probe is studied extensively via microscopy and the photophysical properties compared w.r.t to the morphological transformations. The AIEE probe has been designed using an unusual pyridothiazole scaffold unlike the commonly used archetypal AIE scaffolds based on tetraphenylethene (TPE) and hexaphenylsilole (HPS) and hence, the work also has implications in designing new generation AIEE dyes based on novel scaffold reported.

File list (1) 


\section{Synthesis and self-assembly studies of a new AIEE probe and its application in sensing amyloid fibrillation}

Nidhi Gour ${ }^{[a]^{*}}$, Vivekshinh Kshtriya ${ }^{[a]}$, Shradhey Gupta ${ }^{[b]}$, Bharti Koshti ${ }^{[a]}$, Ramesh Singh $^{[b]}$, Dhaval Patel ${ }^{[c]}$, Khashti Ballabh Joshi ${ }^{[b]^{*} \text {, }}$

[a]Department of Medicinal Chemistry, Indian Institute of Advanced Research, Gandhinagar,

Gujarat, 382426, India; E-mail: nidhi.gour@iar.ac.in; gournidhi@gmail.com Fax: +91 79

30514110. Tel: +91 7930514143

[b] Department of Chemistry, School of Chemical Science and Technology, Dr. Harisingh Gour, Central University, Sagar, India;

E-mail: kbjoshi77@gmail.com; kbjoshi@dhsgsu.ac.in

[d] Department of Bioinformatics and Structural Biology , Indian Institute of Advanced Research, Gandhinagar,dhaval@iiar.res.in; pateldhaval.in@gmail.com 
Abstract: We report self-assembly and photophysical properties of a new pyridothiazole based aggregation-induced-emission enhancement (AIEE) luminogen 4-(5-methoxy-thiazolo[4,5b]pyridin-2-yl)benzoic acid (PTC1) and its application for the sensitive detection and monitoring of amyloid fibrillation. The self-assembling properties of the new AIEE probe are extensively studied by AFM and it was noted that as aggregation increases there is enhancement of fluorescence. The fluorescence of PTC1 is quenched upon addition of cupric $\left(\mathrm{Cu}^{2+}\right)$ ions while the fluorescence is regenerated in the presence of amyloid fibers. AFM studies reveal that PTC1 self associate/aggregate to hairy micelle structures which gets disrupted on the addition of $\mathrm{Cu}^{2+}$ and again reassembles in the presence of amyloid fibers. Hence, the fluorescence quenching and regeneration may be attributed to disaggregation and AIE respectively. Further, a comparative analysis of the performance of PTC1 is done with the conventional ThT which confirms it to be a more sensitive probe for the detection of amyloid both in the presence and absence of $\mathrm{Cu}^{2+}$. Of note, a very simple, facile and cost-effective methodology for the detection of amyloid fibres is presented, wherein fluorescence quenching/enhancement can be visualized under UV without the use of sophisticated instrumentation techniques. To the best of our knowledge and literature survey, this is first report wherein the self-assembling properties of AIEE probe is studied extensively via microscopy and the photophysical properties compared w.r.t to the morphological transformations. The AIEE probe has been designed using an unusual pyridothiazole scaffold 
unlike the commonly used archetypal AIE scaffolds based on tetraphenylethene (TPE) and hexaphenylsilole (HPS) and hence, the work also has implications in designing new generation AIEE dyes based on novel scaffold reported.

Keywords: Amyloid sensing; Aggregation Induced Emission Enhancement (AIEE); Atomic force microscopy; Fluorescence Turn OFF/ON; Molecular docking and simulation

\section{Introduction}

A major challenge in the Alzheimer's disease (AD) is its timely diagnosis. ${ }^{1-2,}$ Main hallmarks of this disease is the aggregation of protein due to misfolding. Amyloid $\beta(A \beta)$ aggregates have been proposed as the most feasible biomarker for the diagnosis of $\mathrm{AD}$ as the neuronal plaques are mainly formed by the aggregation of $\mathrm{A} \beta_{42}$ peptide which is known to induce a range of cytotoxicities. ${ }^{3,4}$ One of the major obstacles in the diagnosis and detection of AD is the lack of effective methodologies that could sense amyloid aggregates timely and selectively. The results of amyloid binding assays known so far in literature are not infallible and often produce false positives/negatives. ${ }^{5-7}$ Thioflavin $\mathrm{T}$ is the most commonly used dye for sensing amyloid fiber however its application is also limited by weak fluorescence and sensitivity. ${ }^{8,9}$ These drawbacks of the conventional fluorophores may be rectified by new class of molecules exhibiting aggregation induced emission (AIE) or Aggregation induced emission enhancement (AIEE). ${ }^{10-12}$ The main difference between AIE and AIEE probe is AIE probe remain non fluorescent in molecular dissolved state while AIE probe is fluorescent under dissolved state too and it fluorescence 
increasesas aggregation increases. ${ }^{12}$ AIE/AIEE probes have many advantages over conventional fluorophore since it remains unaffected by quenching which occurs at a higher concentration or prolonged incubation. ${ }^{13}$ In conventional fluorophores, aggregation causes quenching (ACQ) at higher concentrations, however, in AIE/AIEE probes, aggregation leads to remarkable enhancement of fluorescence along with exceptionally good signal-to-noise ratio. ${ }^{14}$ The most common and typical examples of AIE probes are tetraphenylethene (TPE) ${ }^{15}$ and hexaphenylsilole $(\mathrm{HPS})^{16}$, like propeller or rotor molecules AIE arises in these probes by the restricted twisting or rotation caused by aggregation which leads to stabilization of locally excited (LE) state. ${ }^{17}$ Several literature reports have demonstrated the excellent application of the use of AIE/AIEE probes in biosensing, for the detection and monitoring of plethora of disease biomarkers and as theranostic tool. ${ }^{18-24}$ Recent reports also suggest that AIE probes could detect amyloids with high sensitivity as compared to conventional ThT due to their excellent quantum yield and resistance to quenching ${ }^{25-27}$ Herein, we report the facile synthesis and photophysical properties of the new pyridothiazole construct 4-(5-methoxythiazolo[4,5-b]pyridin-2-yl)benzoic acid (PTC1) which exhibits aggregation-induced emission enhancement (AIEE) properties and its application as a sensor for sensitive detection of amyloid fibers. The fluorescence of the new probe is quenched in the presence of $\mathrm{Cu}^{2+}$ while a significant recovery in fluorescence (turn-ON) can be visualized on the addition of amyloid fibers. PTC1 has a tendency to self-associate and aggregation leads to photoluminescence. When $\mathrm{Cu}^{2+}$ is added the aggregates are disrupted and hence the fluorescence is quenched while upon addition of amyloid re-aggregation occurs which leads to the 
regeneration of fluorescence (Fig.1). Notably, the fluorescence turn-OFF/ON can be visualized with the naked eye under UV chamber without the use of sophisticated instrumentation hence a very simple, rapid and cost-effective methodology for the detection of amyloid is presented.

\section{Figure 1}

\section{Experimental Section}

Materials and Chemicals: All the starting materials for PTC1 synthesis were obtained from commercial suppliers and used as received. 2-Amino-6-methoxypyridine and Benzoyl isothiocyanate were purchased from Combi-Blocks, USA. Acetone, THF, Lithium bromide, Lithium hydroxide, ethyl acetate, hexane, N, N dimethyl formamide (DMF), sodium hydroxide, sodium bicarbonate, sodium sulphate was purchased from Sisco Research Laboratories (SRL), India. Sodium hydroxide, Bromine $\left(\mathrm{Br}_{2}\right)$, Tert-Butyl nitrite, 4-Bromo phenyl acetate, $\mathrm{Cu}(\mathrm{I}) \mathrm{Br}$, Palladium(II)Acetate and Xantphos was from Spectrochem, India. Acetic acid and Toluene were from Finar, India while $\mathrm{HCl}$ was from Merck. Moisture sensitive reactions were performed under an atmosphere of dry Nitrogen. All the solvents used for reactions were distilled prior to use. The water used for all microscopy and spectroscopy measurement was ultra pure water obtained from Millipore Direct-Q ${ }^{\circledR} 5$ UV Water Purification System. A $\beta$ (1-42) fragment, $A \beta$ (1-40) fragment, diphenylalanine (FF), were purchased from Sigma (USA). Thioflavin T (ThT) was purchased from sigma and further recrystallized prior to use. Column chromatography was carried out on silica gel (200-300 mesh). The $\mathrm{R}_{\mathrm{f}}$ was recorded in Analytical TLC Silica Gel $60 \mathrm{~F}_{254}$ purchased from Merck (Germany). The melting point of various intermediates and PTC1 was recorded in Visual Melting Range Apparatus (MR-VIS) provided by LABINDIA. ${ }^{1} \mathrm{H}$ NMR (400 MHz) and ${ }^{13} \mathrm{C}$ NMR (100 MHz) spectra were recorded on Avance III 400 NMR spectrometer instrument. 
Proton chemical shifts are reported in parts per million. HPLC was done using Waters E2695 machine. HPLC was performed using ammonium bicarbonate buffer (ABC) 27 minutes run time and water: ACN as mobile phase in C-18 Column. LC-MS was obtained on an Waters 2690 LCMS instrument. The method used was Ammonium bicarbonate Buffer (ABC); 7 minutes run time and water: ACN as mobile phase in C-18 Column. UV-visible spectra were recorded on a LABINDIA UV-Vis Spectrophotometer $3000^{+}$with $10 \mathrm{~mm}$ quartz cell at $25{ }^{\circ} \mathrm{C}$. Fluorescence spectra were recorded on RF-5301PC Spectrofluorophotometer (Shimadzu) with a $1 \mathrm{~mm}$ quartz cell at $25{ }^{\circ} \mathrm{C}$. Fluorescence lifetime was measured by single photon counting method using a commercial setup from Jobin-Yvon. The samples were excited at appropriate wavelength using a nano-LED. Stock solution of PTC1 were prepared by making 20mM PTC1 solution in DMSO. Subsequently, 1mM stock solution was prepared by dissolving 50 $\mu \mathrm{L}$ of PTC1 in 1mL water.

Synthesis of PTC1: The pyridothiazole construct (PTC1) was synthesized by using the standard synthetic methodology in 6 steps starting from 2-amino-6-methoxy pyridine (See ESI for more detail). To introduce a thiazole moiety, first, the amino group of 2-amino-6-methoxy pyridine was coupled with benzyl isothiocyanate followed by hydrolysis leading to the formation of a thiourea derivative of 6-methoxy pyridine. Next intramolecular cyclization was carried out in the presence of lithium bromide and bromine by using acetic acid as a solvent to yield a fused thiazole ring. In the next step, deamination was carried out via previously reported methodology which is necessary for further $\mathrm{C}-\mathrm{C}$ coupling. Further, $\mathrm{C}-\mathrm{H}$ activation was done using palladium acetate and Xantphos and C-C coupling were done with 4-bromophenyl acetate which upon subsequent hydrolysis yielded PTC1 (ESI). 
Fluorescence spectroscopy of PTC1: The Excitation and Emission spectra of PTC1 were recorded at $10 \mu \mathrm{M}$ concentration in deionized water. The emission spectra of PTC1 was recorded by giving excitation wavelength as $340 \mathrm{~nm}$ and emission was recorded in range 350-600 nm keeping slit width 3. Similarly, excitation spectra were recorded in range 250-600 nm using emission wavelength of $418 \mathrm{~nm}$.

Fluorescence spectroscopy measurements of the interaction of PTC1 with $\mathbf{C u}^{2+}: 10 \mu \mathrm{L}$ of PTC1 (1mM) was added to $990 \mu \mathrm{L}$ DI water and fluorescence of PTC1 alone was recorded at $10 \mu \mathrm{M}$ concentration. PTC1 was added always fresh before the assay to prevent unwanted aggregation. To assess PTC1 interaction with $\mathrm{Cu}^{2+}$ increasing concentration of $\mathrm{Cu}\left(\mathrm{NO}_{3}\right)_{2} 2.5 \mathrm{H}_{2} \mathrm{O}$ was added from the stock solution $(100 \mathrm{mM})$ from $1 \mu \mathrm{L}-20 \mu \mathrm{L}(100 \mu \mathrm{M}-2 \mathrm{mM})$. The concentration of PTC1 was kept fixed at $10 \mu \mathrm{M}$.

Preparation of stock solution of $\mathbf{A} \boldsymbol{\beta}_{42}$ fibrils: $A$ fresh stock solution of concentrated $A \beta-42$ peptide was prepared by dissolving $1 \mathrm{mg}$ of the lyophilized peptide into $440 \mu \mathrm{L}$ of anhydrous DMSO. Then, a working solution of $A \beta_{42}$ was prepared for a fibrillation study by diluting $125 \mu \mathrm{l}$ of peptide solution in a $5 \mathrm{~mL}$ buffer solution at $\mathrm{pH} 2.0$ by adding $\mathrm{HCl}$. Additionally; the solution had $\mathrm{NaCl}(137 \mathrm{mM})$ and $\mathrm{KCl}(2.7 \mathrm{mM})$. The peptide solution was then incubated at $37^{\circ} \mathrm{C}$ for 15 days to achieve complete fibrillation.

Fluorescence spectroscopy analysis of PTC1 assay with Phe-Phe (FF) and FF:Tannic acid: $50 \mu \mathrm{L}$ of PTC1 was taken from $1 \mathrm{mM}$ stock solution and $20 \mu \mathrm{L} \mathrm{Cu}\left(\mathrm{NO}_{3}\right)_{2} 2.5 \mathrm{H}_{2} \mathrm{O}(100 \mathrm{mM})$ was added. Subsequently $400 \mu \mathrm{L}$ FF (1mM) and $400 \mu \mathrm{L}$ FF with Tannic acid (1mM:1mM) were added The final volume of the solutions was adjusted to $1000 \mu \mathrm{L}$ with DI water. The fluorescence spectra were recorded by diluting the sample 5 times. 
PTC1 interaction with amyloid fibrils $(0.5 \mu \mathrm{M})$ in absence of $\mathrm{Cu}^{2+}$ : A stock solution of PTC1 $(1 \mathrm{mM})$ was prepared in deionised water, and $5 \mu \mathrm{L}$ of this solution was mixed with $10 \mu \mathrm{L}$ of $\mathrm{A} \beta_{42}$ peptide (15 days aged solution) and solution was adjusted to final volume upto $500 \mu \mathrm{L}$ by Tris buffer $\mathrm{pH}$ 7.4. The fluorescence was measured under $340 \mathrm{~nm}$ excitation.

PTC1 interaction with amyloid fibrils $(0.5 \mu \mathrm{M})$ in presence of $\mathrm{Cu}^{2+}$ : A stock solution of PTC1 $(1 \mathrm{mM})$ was prepared in deionised water, and $5 \mu \mathrm{L}$ of this solution was mixed with $10 \mu \mathrm{L}$ of $\mathrm{A} \beta-42$ peptide (15 day aged solution) and $2.5 \mu \mathrm{L} \mathrm{Cu}\left(\mathrm{NO}_{3}\right)_{2} .5 \mathrm{H}_{2} \mathrm{O}(100 \mathrm{mM}$ stock) solution was adjusted to final volume of $500 \mu \mathrm{L}$ by Tris buffer $\mathrm{pH}$ 7.4. The fluorescence was measured under $340 \mathrm{~nm}$ excitation.

Sequential regeneration of fluorescence of PTC1- $\mathrm{Cu}^{2+}$ complex by amyloid A $\beta-42$ : A stock solution of PTC1 (1 mM) was prepared in deionised water, and $5 \mu \mathrm{L}$ of this solution was mixed with $10-150 \mu \mathrm{L}$ of $\mathrm{A} \beta-42$ peptide (15 day aged solution) and $5 \mu \mathrm{L} \mathrm{Cu}\left(\mathrm{NO}_{3}\right)_{2} .5 \mathrm{H}_{2} \mathrm{O}(100 \mathrm{mM}$ stock) solution was adjusted to final volume of $500 \mu \mathrm{L}$ by $20 \mathrm{mM}$ Tris buffer $\mathrm{pH}$ 7.4. The fluorescence was measured under $340 \mathrm{~nm}$ excitation.

\subsection{Fluorescence spectroscopy analysis of PTC1 interaction with heat induced amyloid in} BSA in presence of $\mathrm{Cu}^{2+}: 50 \mathrm{mg} / \mathrm{mL}$ BSA was dissolved in DI water and heated at $75^{\circ} \mathrm{C}$ and $85^{\circ}$ C for 10 minutes. The sample of BSA were kept in eppendorf thermomixer at $950 \mathrm{rpm}$ shaking. Subsequently to $980 \mu \mathrm{L} \mathrm{BSA}, 10 \mu \mathrm{L}$ of PTC1 (from stock: $1 \mathrm{mM}$ ) and $5 \mu \mathrm{L} \mathrm{Cu}^{2+}$ (from $100 \mathrm{mM}$ $\mathrm{Cu}\left(\mathrm{NO}_{3}\right)_{2} .2 .5 \mathrm{H} 2 \mathrm{O}$ was added.

Monitoring of amyloid fibrillation by PTC1 assay in presence of $\mathrm{Cu}^{2+}$ : PTC1 binding assay was done by collecting aged $A \beta$ peptide solution- incubated for different time periods- and 
mixing them with PTC1, $\mathrm{Cu}^{2+}$ and $20 \mathrm{mM}$ Tris buffer $\mathrm{pH}$ 7.4. $50 \mu \mathrm{L}$ of $\mathrm{A} \beta$ peptide/fibril solution (aged for $1,2,3,5,7,10,12$ and 15 days) was added with $2.5 \mu \mathrm{L} \mathrm{Cu}\left(\mathrm{NO}_{3}\right)_{2} .5 \mathrm{H}_{2} \mathrm{O}$ and $10 \mu \mathrm{L}$ PTC1. The volume this solution was adjusted to $500 \mu \mathrm{L}$ by Tris buffer $\mathrm{pH}$ 7.4. The sample was incubated for 5 mins at room temperature and the fluorescence was measured under $340 \mathrm{~nm}$ excitation.

ThT binding assays: In the case of the Thioflavin T probe, a stock solution of ThT $(400 \mu \mathrm{M})$ was prepared in a $20 \mathrm{mM}$ Tris buffer at $\mathrm{pH} 7.4$, and $50 \mu \mathrm{L}$ of this solution was mixed with $5 \mu \mathrm{L}$ of $\mathrm{A} \beta$ peptide solution (aged for 15 days) and solution was adjusted to $500 \mu \mathrm{L}$ by Tris buffer $\mathrm{pH}$ 7.4.. After 5 min of incubation, the fluorescence of ThT was measured under $450 \mathrm{~nm}$ excitation. For studies in the presence of $\mathrm{Cu}^{2+}, 2.5 \mu \mathrm{L} \mathrm{Cu}\left(\mathrm{NO}_{3}\right)_{2} \cdot 5 \mathrm{H}_{2} \mathrm{O}(100 \mathrm{mM}$ stock) solution was added additionally. For monitoring amyloid fibrillation, $50 \mu \mathrm{L}$ of $\mathrm{A} \beta$ peptide/fibril solution (aged for 1 , 2, 3, 5, 7, 10, 12 and 15 days) was mixed with $50 \mu \mathrm{L}$ of ThT stock solution in presence or absence of $5 \mu \mathrm{L} \mathrm{Cu}\left(\mathrm{NO}_{3}\right)_{2} .5 \mathrm{H}_{2} \mathrm{O}$.

Fluorescence life time measurements: Fluorescence lifetime was measured by single photon counting method using a commercial setup from Jobin-Yvon. For lifetime measurement, excitation of samples is $340 \mathrm{~nm}$ by Nano-LED and emission is recorded at $420 \mathrm{~nm}$.

Atomic Force Microscopy (AFM): Neat and co-incubated solutions of PTC1, PTC1 with $\mathrm{Cu}^{2+}$ and PTC1 with amyloidogenic peptides were imaged with an atomic force microscope. The samples were placed on freshly cleaved muscovite mica surfaces followed by imaging with an atomic force microscope (INNOVA, ICON analytical equipment, Bruker, operating under the acoustic AC mode (AAC or tapping mode), with the aid of a cantilever (NSC 12(c) from MikroMasch, Silicon Nitride Tip) by NanoDrive ${ }^{\mathrm{TM}}$ version 8 software. The force constant was $2.0 \mathrm{~N} / \mathrm{m}$, while the resonant frequency was $\sim 276 \mathrm{kHz}$. The images were taken in the air at room 
temperature, with the scan speed of 1.5 lines/sec. The data analysis was done using of nanoscope analysis software. The sample-loaded substrates were dried at dust-free space under a 40W lamp for 30 minutes followed by high vacuum drying and subsequently examined under AFM.

Molecular docking studies: The molecular docking was performed using the AutoDockVina ${ }^{28}$ and the necessary input files were prepared using the AutoDock 4.2. ${ }^{29}$ For docking the PDB:2BEG was chosen as the receptor consisting of $5 \mathrm{~A} \beta_{42}$ peptides from the Protein Data Bank ${ }^{30}$ The TT molecule was taken from PubChem (ID: 16953). The TT and PTC1 were converted to sdf format and minimized using mmff94 force field using Steepest Descent method for 1000 steps. The atomic charges of PTC1 were assigned using antechamber with AM1-BCC method. The rotatable bonds were kept by default rotable and no change was made. A grid box centered on the protein was defined using the dimension of $40 \mathrm{X} 40 \mathrm{X} 40$ using $\mathrm{XYZ}$ coordinates $(-7.593,0.520,-8.532)$ and 0.375 A grid step. The grid size and dimension was chosen in such a way that plausible amino acids are covered in the probable site which is proposed in earlier studies. ${ }^{31}$ The same coordinates for $\mathrm{A} \beta_{42}$ peptides were used for both ThT and PTC1. Vina uses iterated local search global optimizer algorithm for docking calculations. All the parameters for vina run were set as default except the exhaustiveness for the search was set to 8.

Molecular Dynamics Simulations (MDS): To access the binding stability of PTC1 in comparison to ThT with the $\mathrm{A} \beta_{42}$ and for validation of molecular docking, the best docking pose was selected as initial conformation for $5 \mathrm{~ns}$ molecular dynamics simulations (MDS) as employed earlier. ${ }^{32} \mathrm{~A}$ total of three simulations system were generated: one for apo-protein $\left(\mathrm{A} \beta_{42}\right)$, $\mathrm{A} \beta_{42}$ in complex with ThT $\left(\mathrm{A} \beta_{42}\right.$ ThT) as a reference compound and $\mathrm{A} \beta_{42}$ in complex with PTC1 
(A $\beta_{42}$ PTC1). For all the MDS, the structure for $A \beta_{42}$ was taken from the Protein Data Bank (PDB:2BEG). $34^{42}$ MDS was carried out in GROMACS 2018 version $^{35}$ using GROMOS96-43a1 force-field. For inhibitor topology and parameter generation, PRODRG tool was used. ${ }^{36}$ All the MDS system were solvated using with Simple Point Charge (SPC) water model in dodecahedron box maintaining a distance of $1 \mathrm{~nm}$ from all the directions of protein and periodic boundary. As the net charge of $A \beta_{42}$ is -5 , an equal number of $\mathrm{Na}^{+}$counterions were added for neutralization of systems. In order to remove steric clashes and bad contacts, all the MDS systems were subjected to energy minimization using the steepest descent algorithm to generate maximum force below $1000 \mathrm{~kJ} \cdot \mathrm{mol}^{-1} \mathrm{~nm}^{-1}$ (50,000 steps max.). After energy minimization, the systems were equilibrated with position restraint simulation of $1 \mathrm{~ns}$ carried out under NVT (the constant Number of particles, Volume and Temperature) and NPT (the constant Number of particles, Pressure and Temperature) conditions. Briefly, the first equilibration was carried at a constant volume for $100 \mathrm{ps}$ at a constant temperature of $300 \mathrm{~K}$ using a modified Berendsen thermostat algorithm. Subsequently, the second equilibration was carried out for 100 ps at a constant pressure of 1 bar maintained by Parrinello-Rahman barostat (Parrinello, 1981). Finally, all the MDS were subjected to $5 \mathrm{~ns}$ production dynamics saving the coordinates at every 2 fs time-step with all the other parameters similar as described above. Finally, the trajectories were visualized by $\mathrm{VMD}^{37}$ and Chimera. ${ }^{38}$ The root mean square deviation (RMSD) and Radius of gyration (Rg) were calculated from final trajectories using rms, gyrate and GRACE (xmgrace) was used for generating and visualization of plots (http:// plasma-gate.weizmann.ac.il/Grace). The binding 
energy of $\mathrm{A} \beta_{42}$ with PTC1 and ThT complex was predicted by free energy calculations. The widely used approach for calculations of free energy binding is Poisson-Boltzmann or generalized Born and surface area continuum solvation (MM-PBSA and MM-GBSA) methods. ${ }^{39}$, The g_mmpbsa tool with all the default parameters ${ }^{40}$ was used for molecular mechanics potential energy (electrostatic + van der waals interactions) and free energy solvation (polar + non-polar solvation energies) calculations. The binding free energy was calculated for an entire trajectory of $5 \mathrm{~ns}$, with a total of 50 frames with the time frame of 100 ps in order to encompass different conformational states of the complexes.

\section{Results and Discussion}

The new pyridothiazole construct (PTC1) was synthesized by using standard organic synthesis protocols in 6 steps from 2-amino-6-methoxy pyridine $\left(\mathrm{ESI}^{\dagger}\right)$ followed by the structural characterization. The synthetic procedure for preparing PTC1 is illustrated in scheme 1. To design PTC1 the basic skeleton of the molecular rotor ThT was retained, but herein we replaced the benzothiazole moiety by methoxy substituted pyridothiazole group, a well-known metal chelator. Previously, many charge transfer based fluorescence switch systems were synthesized using pyridothiazole moiety by varying its electron releasing and withdrawing group, hence this modification was made. ${ }^{41}$ The insertion of electron withdrawing group i.e. $-\mathrm{COOH}$ group in 
PTC1 instead of electron donating group (N, N-dimethylamino) of ThT was done to ensure different photophysical properties of PTC1. It was envisaged that an electron donating methoxy group on pyridothiazole and electron withdrawing group on another side of the rotor will cause restricted rotation and push PTC1 towards locally excited (LE) state rather than twisted intramolecular charge transfer (TICT) which exist in ThT. Interestingly, it could be deciphered by TLC spots while synthesis, that PTC1 exhibits good fluorescence under UV chamber.

\section{Scheme 1}

The photophysical properties of PTC1 were assessed by first taking its UV visible absorption spectra (Fig. S1, ESI) which revealed the absorption maximum at around $350 \mathrm{~nm}$. Further, the fluorescence spectra of PTC1 reveal excitation maximum at $337 \mathrm{~nm}$ and emission maximum at around $420 \mathrm{~nm}$ (Fig.2A). Interestingly, the fluorescence of PTC1 can be visualized with the naked eye under UV Chamber and intense blue emission could be assessed in a vial containing PTC1 while ThT did not reveal any such fluorescence (Fig. S2, ESI). The emission spectra of PTC1 indeed revealed that the fluorescence of the new probe is exceptionally good with high signal to noise ratio. Since the structure of PTC1 was analogous to ThT, a relatively low quantum yield fluorescence probe, it was surprising that PTC1 revealed such exceptionally high 
fluorescence (Fig 2A). Hence, we were motivated to study the aggregation properties of PTC1 probe since it's reported that aggregation of the molecule leads to the restricted intramolecular rotation(RIR) and yield high fluorescence. ${ }^{42-44}$ Accordingly, AFM studies were pursued to investigate the self-assembly of PTC1. The AFM studies of PTC1 revealed that it assembles into cylindrical hairy micelles or supermicelles like structures with small fibers protruding from sides of micelle (Fig.2B). Such micelle morphology also indicated that PTC1 will have a tendency to self-aggregate further and assembled into febrile structures. Prolonged aggregation studies indeed revealed the formation of mature fibers (5 days, Fig.2C) which get converted into twisted fibers like morphology when incubated for 15 days (Fig.2D). Hence, it was evident that the aggregation of PTC1 might play a crucial role in its exceptional fluorescence. In addition, PTC1 also revealed other characteristic features of AIEE probes i.e. a continuous increase in emission intensity could be assessed with respect to time and concentration. The fluorescence of the conventional fluorophores, unlike AIEgen, quenches with prolonged incubation periods and concentration due to aggregation. The aggregation of PTC1 may be mainly attributed to pi-pi stacking interactions along with hydrogen bonding as in other AIE probes. ${ }^{45-47}$

\section{Figure 2}


Further, a solvent dependent study was done to understand the effect of solvent polarity in aggregation of PTC1.For these studies PTC1 aggregation studies were done in a mixture of tetrahydrofuran (THF) and water. While water is polar in nature, THF is a non polar solvent. Hence this binary mixture is ideal for studying self-assembly of amphiphilic molecules and it was expected that the addition of THF will further induce aggregation of PTC1. ${ }^{44}$ Interestingly, as the ratio of water increased in THF/water mixture, there was increase in fluorescence. AFM micrograph of 1:9 water:THF revealed micellar structures with fibers protruding outside while AFM micrograph of 9:1 water:THF revealed dense fibrillar tape-like aggregates. From the AFM micrograph and the corresponding fluorescnce, it could be surmised that the aggregation of PTC1 was effected by the solvent polarity and the fluorescence increases with aggregation (Fig. 3). Hence, PTC1 clearly exhibits aggregation-induced-emission characteristics.

\section{Figure 3}

Since PTC1 also has pyridine moiety which acts as an effective metal chelator, we were also curious to investigate the effect of the interaction of metal ions with PTC1 and visualize its effect on the PTC1 fluorescence property. Metal ions are also reported to be very effective fluorescence quenchers. ${ }^{48}$ Since the basic idea of designing PTC1 was to assess its application in amyloid sensing, we wanted to first assess the interaction of PTC1 with metals like $\mathrm{Cu}$ and $\mathrm{Zn}$ as they are known to have a high affinity for amyloids and many metal-assisted amyloid fibrillation are 
reported with these metal ions. ${ }^{49}$ As expected, the fluorescence of PTC1 was quenched by both $\mathrm{Cu}^{2+}$ and $\mathrm{Zn}^{2+}$ (Fig. S3, ESI). However, quenching was more effective in the case of $\mathrm{Cu}^{2+}$ hence, the fluorescence emission spectra were recorded using varying concentrations of $\mathrm{Cu}^{2+}$ only (Fig. 4A). AFM micrograph of PTC1 in the presence of $\mathrm{Cu}^{2+}$ revealed that the aggregates/assembly of PTC1 got disrupted on the addition of $\mathrm{Cu}^{2+}$ (Fig. 4B). Prolonged incubation studies of PTC1 were also done with $\mathrm{Cu}^{2+}$ (till 15 days) and notably, the fluorescence signal of the fluorophore PTC1 remains quenched and also no regeneration of aggregates could be assessed (data not shown). Hence the AFM and fluorescence studies of PTC1 with $\mathrm{Cu}^{2+}$ further implicated aggregation plays an important role, in the fluorescence of PTC1 and quenching may be attributed to disaggregation caused by $\mathrm{Cu}^{2+}$. The observed quenching of fluorescence intensity after the addition of $\mathrm{Cu}^{2+}$ ions may be due to photo-induced electron transfer (PET) process and static quenching mechanism, indicating the formation of a complex between $\mathrm{Cu}^{2+}$ and PTC1.$^{50-53}$ The quantitative effect of fluorescence quenching was also determined by plotting Stern-Volmer plot (Fig. S4, ESI). The obtained values of the Stern-Volmer constant for PTC1 in presence of $\mathrm{Cu}^{2+}$ was found to be $1860.7 \times 10^{-6} \mathrm{M}^{-1}$ respectively. The plot between used $\mathrm{Cu}^{2+}$ ions concentration and $\tau_{\mathrm{o}} / \tau$ furnished straight line which hints that the nature of quenching in the present case is static, i.e. $\mathrm{Cu}(\mathrm{II})$ ions directly formed complex with the PTC1 molecular structure (Fig.S4B, ESI). ${ }^{51-53}$ 
Since there was a structural similarity between PTC1 and ThT, it was also expected that PTC1 should have an affinity for binding amyloid fibers. Hence, we were motivated to assess the application of PTC1 as an amyloid sensor and investigate, if the fluorescence of PTC1 which was quenched by $\mathrm{Cu}^{2+}$ could be regenerated by the addition of $\mathrm{A} \beta_{42}$ fibers. $\mathrm{A} \beta_{42}$ mature fibrils were formed by previously reported methodology (ESI). ${ }^{27}$ As $\mathrm{A} \beta_{42}$ fibers were added in increasing concentration to the solution of $\mathrm{Cu}(\mathrm{II})$ coordinated PTC1, a surprising recovery of the quenched fluorescence intensity could be assessed (Fig. 4C). To investigate whether the aggregation properties of PTC1 are also regenerated on the addition of $\mathrm{A} \beta_{42}$, AFM micrograph was recorded. Interestingly AFM revealed re-assembled PTC1 and the micelles of PTC1 could be seen binding to amyloid fibers (Fig. 4D). It may be interpreted that due to the high affinity of PTC1 to A $\beta_{42}$ fibers, PTC1 cling to amyloid fibers followed by the self-association and leading to regeneration of fluorescence. Notably, PTC1 binding assays were also effective for detection of other amyloid model like Phe-Phe which is used as reductionist model for $\mathrm{A} \beta_{42}{ }^{20}$ (Fig. S9a, ESI).

\section{Figure4}

It is well known that tannic acid is a very effective amyloid inhibitore and it effectively breaks the amyloid fibrils. The TEM image of amyloid with tannic acid clearly reveals complete break down of amyloid fibrils. Hence we added tannic acid toPTC1-amyloid regenerated system. 
Addition of tannic acid indeed induced fluorescence quenching suggestingstrongly that PTC1 bind to amyloid fibrils and show more enhancement with fibers than monomer. Hence the study clearly revealedutility of PTC1 for monitoring amyloid fibrillation process. We will be studying utility of PTC1 for screening amyloid inhibitors in greater detail in our future work Next, to assess the selectivity of PTC1 binding assay, for amyloid, we took native bovine serum albumin (BSA) as negative control. Native BSA protein does not have beeta sheet however, beEta sheets can be thermally induced in BSA if it is heated upto $85^{\circ} \mathrm{C} . .^{33}$ Figure $4 \mathrm{~A}$ reveals the fluorescence spectra of PTC1-Cu (II) complex with native BSA and heat treated BSA. It may be noted that the addition of native BSA did not induced any recovery of PTC1 fluorescence, however addition of heat treated BSA caused partial recovery. Hence, the study implicates that since BSA acquired $\beta$ sheet structures on heating, PTC1 was now able to bind to BSA and hence fluorescence turn-ON could be assessed. The experiment also show that fluorescence is regenerated only if BSA form fibrils and not with native BSA.

\section{Figure 5}

Further,comparative analysis of the performance of ThT and PTC1 for the detection of amyloid was also performed in the presence of amyloid. Interestingly, the fluorescence of PTC1 was enhanced on the addition of 0.5 micromole of $\mathrm{A} \beta_{42}$ (Fig. 6A). ThT, on the other hand, reported no 
fluorescence enhancement at such a low concentration of amyloid. Hence, PTC1 clearly revealed a more sensitive detection of amyloid fibrillation assisted by metal ions wherein ThT show false negative The quantum yield of PTC1 both in presence and absence of amyloid was also exceptionally high as compared to ThT (Fig. 5B). Moreover, PTC1 binding assays were more sensitive that ThT assay even for the detection of other amyloid models- FF (Phe-Phe, dipeptide)which is used as reductionist model for $\mathrm{A} \beta_{42}^{56}$ and $\mathrm{A} \beta_{40}$ (Fig S7, ESI).

\section{Figure 6}

Since $\mathrm{A} \beta_{42}$ monomer transforms from oligomer to protofibril stage followed by fibril formation in a time-dependent fashion, ${ }^{57}$ we also performed a time-dependent fluorescence recovery assay with $\mathrm{A} \beta_{42}$ incubated at different time intervals. The fluorescence which was quenched in fresh condition by cupric ions was gradually recovered during the course of 15 days, implicating that PTC1 binding affinity for $\mathrm{A} \beta$ increases as it gradually changes from oligomer to fibril stage. A conventional ThT binding assay was also done under similar conditions in presence of $\mathrm{Cu}^{2+}$ and addition of amyloid aged at different time intervals. Surprisingly there was no enhancement in fluorescence of $\mathrm{ThT}$ in presence of $\mathrm{Cu}^{2+}$. The fluorescence enhancement which was expected to occur on the binding of ThT to amyloid fibers could not be seen in the presence of $\mathrm{Cu}^{2+}$. Hence a false negative result was obtained via ThT binding assay in presence of $\mathrm{Cu}^{2+}$. As compared to ThT, PTC1 binding assay showed significant recovery of fluorescence and it could be surmised 
that PTC1 is a more effective probe for sensing amyloid as compared to ThT. Even in the absence of $\mathrm{Cu}^{2+}$ ions, the fluorescence intensity of ThT binding assay was very less as compared to PTC1. The experiment henceforth clearly revealed superior detection properties of PTC1 binding assay as compared to ThT binding assay and confirmed that PTC1 is a better alternative probe to ThT for detection of amyloid fibrillation (Fig. 5C). The fluorescence lifetime of PTC1 was also very high (3.9 ns, Fig. 5D) as compared to that reported for ThT (1ps). ${ }^{85}$ Hence, PTC1 clearly exhibits superior photophysical properties as compared to ThT and it is potentially a better probe. The photophysical properties of PTC1 are summarized in (Table 1, ESI).

Additionally, to assess the binding and interaction of PTC1 with $\mathrm{A} \beta_{42}$, theoretically, we performed docking studies of PTC1 and ThT with amyloid fibrils obtained from the protein database (PDB code: 2BEG). The results suggested that PTC1 can only be docked to the flanks of the fibril i.e. particularly to the entry cleft site and not into the core sites. However, our results also suggest that PTC1 is able to dock more tightly in the entry flanking region compared to ThT as shown in figure 6(A). Like the fact that aromatic amino acids are very crucial to the interaction of ThT to $\mathrm{A} \beta_{42}$, we found similar results that in both the cases -ThT and PTC1-, Phe19 plays an important role as assessed by figure $6(\mathrm{~B}, \mathrm{C})$. The docking empirical score for the PTC1 was found to be $-6.6 \mathrm{kcal} / \mathrm{mol}$ which is higher than that of ThT $(-6.2 \mathrm{kcal} / \mathrm{mol})$.

\section{Figure 6}


Further, the Molecular Dynamics Simulations (MDS) were also carried out to study the conformational stability and prediction of binding free energy in complex formed by $\mathrm{A} \beta_{42}$-PTC1 and $\mathrm{A} \beta_{42}$-ThT. In the present study, we analyzed the RMSD, gyrate as well as the binding free energy of the complexes and compared with apo-protein's MDS. In order to assess the dynamic stability of $\mathrm{A} \beta_{42}, \mathrm{~A} \beta_{42}$-ThT, $\mathrm{A} \beta_{42}$-PTC1, RMSD values for $\mathrm{C}_{\alpha}$ backbone were calculated for the entire $5 \mathrm{~ns}$ simulations. RMSD is a means to measure the structural variation between the $\mathrm{C}_{\alpha}$ back-bone from its initial conformation to its final position during the entire simulation trajectory. Smaller RMSD values indicate higher stability of the simulation. The RMSD values are shown in the plot of RMSD (nm) vs. Time (ns) in figure 7A. The average RMSD values with S.D. for A $\beta_{42}$, $\mathrm{A} \beta_{42^{-}}$ThT, A $\beta_{42^{-}}$PTC1 were 0.29 (0.040), 0.30 (0.034) and $0.22(0.024) \mathrm{nm}$ respectively. The RMSD analysis revealed that the A $\beta_{42}$ PTC1 was much more stable in comparison to the apo and $\mathrm{A} \beta_{42}$-ThTreference MDS. The radius of gyration $\left(\mathrm{R}_{\mathrm{g}}\right)$ is a measure of how compact is the protein fold during the course of the simulation. The stable values of $R_{g}$ indicate a stable fold during the MDS and unfolded state will have varying $R_{g}$ values over the time. The $R_{g}$ values are shown in the plot of $R_{g}(n m)$ vs. Time (ns) in figure 7B. The average $R_{g}$ values with S.D. for $A \beta_{42}$, $\mathrm{A} \beta_{42}$-ThT, and $\mathrm{A} \beta_{42}$-PTC1 was $1.37( \pm 0.025), 1.39( \pm 0.015)$ and $1.40( \pm 0.009) \mathrm{nm}$ respectively. From the $\mathrm{R}_{\mathrm{g}}$ graph, it is clearly evident that the apo-protein $\left(\mathrm{A} \beta_{42}\right)$ has varying $\mathrm{Rg}$ values as compared to the $\mathrm{A} \beta_{42}$-ThT and $\mathrm{A} \beta_{42}$-PTC1. These stable $\mathrm{R}_{\mathrm{g}}$ values over the time indicate that there is stable complex formation maintaining the fold and interestingly, for $\mathrm{A} \beta_{42}$-PTC1 the $\mathrm{R}_{\mathrm{g}}$ 
values have the least deviation indicating a better complex formation. The binding free energy $(\Delta \mathrm{G})$, for both the complexes, was calculated using the MM-PBSA method for entire 5 ns trajectories. The $(\Delta \mathrm{G})$ is an estimation of the non-bonded interaction energies and a total of 50 frames at every 100ps were taken for the calculations. The estimated value of $(\Delta \mathrm{G})$ calculated for $\mathrm{A} \beta_{42}$-ThT and $\mathrm{A} \beta_{42}$-PTC1 were $-165.982 \pm 3.743$ and $-125.675 \pm 1.626 \mathrm{KJ} \mathrm{mol}^{-1}$ respectively. Details of energy contribution from each component are shown in figure 7C. It is a well-known fact that the ThT binds to diverse fibrils irrespective of the primary sequence of them which strongly indicates that the ThT recognizes a common structural feature amongst the fibrils. ${ }^{58}$ Earlier reports have hypothesized that the amyloid interactions by ThT are a result of hydrophobic interactions at the surface of the cross- $\beta$ architecture of fibrils. It is known that the ThT do not bind uniformly to the $\beta$-sheet surface but binds at shallow groove channels formed by aromatic residues (for instance, a Val-Phe cross-strand motif and contacts with the adjacent Leu ladder). The primary reason for ThT binding is because the aromatic residues present a large hydrophobic surface and the capacity to $\pi$-stack with the dye. ${ }^{59-61,}$ Similar results were obtained in our MDS for both the dyes ThT and PTC1. MDS in case of PTC1reveals, the molecule was almost in a similar location with hydrophobic interaction with the aromatic residues (Phe) and interaction with Leu and Val (Figure 7D) as identified earlier with ThT. ${ }^{26}$ The binding free energy estimation for each component (Fig. 7C) also confirmed the increase in Van der Waal interactions in case of PTC1 compared to ThT. Overall, our MDS results indicated that the PTC1 have much more stable interaction (though the binding constant of is less than $\mathrm{A} \beta_{42}$-ThT) 
with the $A \beta_{42}$ compared to ThT with similar binding free energy estimates. From computational studies it was also evident that, though the binding constant of $\mathrm{A} \beta_{42}$-PTC1 was less than $\mathrm{A} \beta_{42^{-}}$ ThT, the binding was more stable and the affinity of PTC1 for amyloid was stronger due to which PTC1 could also potentially displace ThT (used in excess) and the fluorescence could be recovered with the same intensity even in presence of competing ThT probe (Fig. S8, ESI)

\section{Figure7}

Since the simulations suggested that PTC1 has an affinity for binding to amyloid fiber, we also

performed a binding assay of PTC1 to amyloid to get an experimental proof for PTC1-amyloid binding. To quantify the intermolecular interactions between PTC1 and amyloid, the variation in the fluorescence intensity with fibril concentration is fitted with the following modified BenesiHildebrand equation for 1: 1 complexation. $^{62}$

$$
f_{i}=\frac{f_{i}^{o}+f_{i}^{\infty} K_{b}[\text { Amyloid }]}{1+K_{b}[\text { Amyloid }]}
$$


Where $f_{i}$ is the observed fluorescence intensity, $f_{i}^{o} \& f_{i}^{\infty}$ are the fluorescence intensities of free and entirely bound PTC1 with amyloid, respectively. $K_{b}$ is the binding constant. $f_{i}^{\infty} \& K_{b}$ were estimated by fitting the fluorescence data using the above equation. A good correlation $\left(\mathrm{R}^{2}=\right.$ 0.89) between the fitted and fluorescence experimental data plainly assist the single mode of binding between PTC1 and amyloid fibrils. The estimated binding constant from the plot fitting was found to be $6.63 \times 10^{5} \mathrm{M}^{-1}$, indicating good affinity of PTC1 towards the amyloid fibrils (ESI). The binding constant of PTC1 was found to be much better than that of pico green $\left(2.6 \times 10^{4} \mathrm{M}^{-1}\right)$ and comparable to that of ThT (Fig. 8) ${ }^{63}$ Earlier, it has been reported, that PG is a better probe than ThT since it revealed higher fluorescence enhancement and sensitivity on binding with amyloid. It was further explained that although, PG had weaker binding constant than ThT, only $10 \%$ of bound ThT caused fluorescence enhancement while in the case of PG, most of the PG molecules which are bound to amyloid show fluorescence enhancement and hence better sensitivity. ${ }^{64}$ Similar observation can be noted for PTC1, Though, PTC1 may have weaker association constant with amyloid than ThT, the binding of PTC1 to amyloid is more stable and hence it produced more fluorescence enhancement. owing to its high quantum yield which in turn lead to higher signal to noise ratio as compared to ThT.

\section{Figure8}




\section{Conclusions}

In summary, we report a facile synthesis of novel pyridothiazole based fluorophore, PTC1 which conceptually has a similar structure with ThT but exceptionally high quantum yield and fluorescence lifetime. Fluorescence of PTC1was attributed to its aggregation propensity which resulted in AIE. Due to AIE the QY and signal to noise ratio of PTC1 were very high and no quenching of fluorescence occurred over prolonged time. PTC1 could effectively detect amyloid fibers in presence of metal ions like $\mathrm{Cu}$ and $\mathrm{Zn}$ wherein $\mathrm{ThT}$ gives a false negative result. The application of PTC1 as an amyloid sensor through fluorescence turn-OFF-ON has been illustrated using different amyloid models. Fluorescence of PTC1 is quenched in presence of $\mathrm{Cu}^{2+}$ (turn-OFF), while the addition of $\mathrm{A} \beta$ aggregates caused recovery of fluorescence (turn-ON). The fluorescence OFF/ON switch can be assessed with the naked eye under UV chamber too. Hence, PTC1 provides a very economical tool to assess the presence of amyloid fibers in solution without the aid of sophisticated instrumentation techniques. The method can potentially be very useful for the detection of amyloids in vitro and may pave the way for an effective diagnostic tool for early-stage detection of $\mathrm{A} \beta$ aggregates which are the biomarker of Alzheimer's 
disease. Future endeavors will include a more rigorous study of PTC1 as labeling agent and amyloid imaging dye and understanding the mechanism of its aggregation in more detail. The free acid group presents in PTC1 offer a facile modification site in its structure and many new generation AIE dyes could be designed using this as scaffold. The efforts will also be pursued to tune PTC1 absorption properties towards visible and near-infrared region for its wider applicability as amyloid sensing dye in vivo.

\section{ASSOCIATED CONTENT}

The supporting information of this manuscript is available and contains detailed synthesis of

PTC1, additional fluorescence with other amyloid models, AFM images, NMR, LCMS and HPLC data.

\section{AUTHOR INFORMATION}

\section{Corresponding Authors}

Dr. Nidhi Gour, Centre of Engineering and Enterprise, Indian Institute of Advanced Research, Gandhinagar, Gujarat, 382426, India; E-mail: nidhi.gour@iar.ac.in; gournidhi@gmail.com Fax: +9179 30514110. Tel: +91 79 30514143; Dr. K. B. Joshi, Department of Chemistry, School of 
Chemical Science and Technology, Dr. Harisingh Gour Central University, Sagar, MP, Dr. Harisingh Gour, Central University, Sagar,, India; E-mail: kbjoshi77@gmail.com; kbjoshi@dhsgsu.ac.in

\section{Author Contributions}

The manuscript was written through the contributions of all authors. All authors have given approval to the final version of the manuscript.

\section{Funding Sources}

This work is supported by DST-SERB India project no. EMR/2016/003186 to NG and CSIRIndia, project no. 02(0238)/15/EMR-II to KBJ.

\section{Conflict of Interest}

The authors declare no competing financial interest.

\section{Acknowledgments}

VKS and BK thank IAR and SERB-DST for pre-doctoral research fellowship respectively. SG and RS thank SERB-DST and CSIR-UGC-India for N-PDF and JRF fellowships respectively. 
SAIF-IISC Bangalore and SICART are thanked for NMR analysis. Our acknowledgment is incomplete without thanking Prof. Ehud Gazit and his team Dr. Dana Laor, Shon Levkovich and Dor Zaguri for the very useful discussions and to Prof. Sandeep Verma (IIT Kanpur) for his constant motivation and invaluable support. Dr. Sukhendu Nath (BARC) is thanked for his valuable suggestions regarding the fluorescence data.

\section{REFERENCES}

[1]. Frisoni, G. B. et al. (2017) Strategic roadmap for an early diagnosis of Alzheimer's disease based on biomarkers. The Lancet Neurol. 16 (8), 661-676.

[2]. Blennow, K., Mattsson, N., scholl, M., Hansson O., Zetterberg, H. (2015) Amyloid biomarkers in Alzheimer's disease. Trends in Pharmacol Sci. 36 (5), 297-309.

[3]. Dag, A., Byron, C., Marios, P., Chaudhuri, K. R., ffytche, D. H. (2017) Cognitive decline in Parkinson disease. Nat Rev Neurol. 13 (4), 217-231.

[4]. Castellani, R. J., Perry, G. (2014) The complexities of the pathology-pathogenesis relationship in Alzheimer disease. Biochem Pharmacol. 88 (4), 671-676.

[5]. Hudson, S. A., Kee, H. E., Carver, T. W. (2009) The thioflavin T fluorescence assay for amyloid fibril detection can be biased by the presence of exogenous compounds. FEBS J. 276 (20), 5960-5972.

[6]. Noormägi, A., Primar, K., Tougu, V., Palumaa, P. (2012) Interference of low-molecular substances with the thioflavin-T fluorescence assay of amyloid fibril. J. Pept Sci. 18 (1), 59-64.

[7]. Liu, K.N., Wang, H.Y., Chen, C.Y., Wang, S. S. (2010) L-Arginine reduces thioflavin T fluorescence but not fibrillation of bovine serum albumin. Amino Acids. 39 (3), 821-829. 
[8]. Cloe A. et al. (2011) The Japanese Mutant $A \beta$ ( $\triangle E 22-A \beta 1-39)$ Forms Fibrils Instantaneously, with Low Thioflavin T Fluorescence: Seeding of Wild-Type A $\beta 1-40$ into Atypical Fibrils by $\Delta$ E22-Aß1-39. Biochemistry. 50 (12), 2026-2039.

[9]. Smith, N. W., Dzyuba, S. V. (2012) Dye-Binding Assays for Evaluation of the Effects of Small Molecule Inhibitors on Amyloid (A $\beta$ ) Self-Assembly. ACS Chem. Neurosci. 3 (11), $807-$ 819.

[10]. Hong, Y., Lam, J. W. Y., Tang, B. Z. (2011) Aggregation-Induced Emission. Chem. Soc. Rev. 40, 5361-5388.

[11]. Ding, D., Li, K., Liu, B., Tang, B. Z. (2013) Bioprobes Based on AIE Fluorogens. Acc. Chem. Res. 46 (11), 2441-2453.

[12]. Mei, J., Leung, N. L. C., Kwok, R. T. K., Lam, J. W. Y., \& Tang, B. Z. (2015) Aggregation-Induced Emission: Together We Shine, United We Soar! Chem. Rev. 115 (21), $11718-11940$

[13]. Hong Y., Jacky W. Y. Lama, Tang B. Z. (2009) Aggregation-Induced Emission: Phenomenon, Mechanism and Applications. Chem. Commun. 4332-4353.

[14]. Gao M., Tang B. Z. (2017) Fluorescent Sensors Based on Aggregation-Induced Emission: Recent Advances and Perspectives. ACS Sens. 2 (10), 1382-1399.

[15]. Zhao Z., Jacky W., Tang B. Z. (2010) Aggregation-Induced Emission of Tetraarylethene Luminogens. Curr. Org. Chem. 14, 2109-2132.

[16]. Zhao Z., Hea B. and Tang B. Z. (2015) Aggregation-induced emission of siloles. Chem Sci. 6, 5347-5365.

[17] Wang M., Zhang G., Zhang D., Zhu D., Tang B. Z. (2010) Fluorescent bio/chemosensors based on silole and tetraphenylethene luminogens with aggregation-induced emission feature. $J$. Mater. Chem. 20, 1858-1867

[18]. Kwok R., Leung C., Lamab J., Tang B. Z. (2015) Biosensing by Luminogens with Aggregation-Induced Emission Characteristics. Chem. Soc. Rev. 44, 4228-4238. 
[19]. Leung, C. W. T., Hong, Y., Chen, S., Zhao, E., Lam, J. W. Y., Tang, B. Z. (2013) A Photostable AIE Luminogen for Specific Mitochondrial Imaging and Tracking. J. Am. Chem. Soc. 135 (1), 62-65.

[20].Gu X, Kwok RTK, Lam JWY, Tang BZ. (2017) AIEgens for Biological Process Monitoring and Disease Theranostics. Biomaterials. 146, 115-135.

[21]. Feng G., Yuan Y., Fang H., Zhang R., Xing B., Zhang G., Zhang D., Liu B. (2015) A light-up probe with aggregation-induced emission characteristics (AIE) for selective imaging, naked-eye detection and photodynamic killing of Gram-positive bacteria. Chem. Commun. 51 (62), 1249012493

[22] Shi, H., Liu, J., Geng, J., Tang, B. Z., Liu, B. (2012) Specific Detection of Integrin $\alpha v \beta 3$ by Light-Up Bioprobe with Aggregation-Induced Emission Characteristics. J. Am. Chem. Soc. 134 (23), 9569-9572.

[23].Gao M., Tang B. Z. (2017) Aggregation-Induced Emission Probes for Cancer Theranostics. Drug Discov Today. 22 (9), 1288-1294.

[24].Han K, Wang SB, Lei Q, Zhu JY, Zhang XZ. (2015) Ratiometric Biosensor for AggregationInduced Emission-Guided Precise Photodynamic Therapy. ACS Nano. 9 (10), 10268-10277.

[25] Zhang JD, Mei J, Hu XL, He XP, Tian H. (2016) Ratiometric Detection of $\beta$-Amyloid and Discrimination from Lectins by a Supramolecular AIE Glyconanoparticle. Small. 12 (47), 65626567.

[26] Wang, Y.-L., Fan, C., Xin, B., Zhang, J.-P., Luo, T., Chen, Z.-Q., Zhou Q.-Y., Yu, Q., Li, X.-L., Huang Z.-L., Li, C.,Zhu M.-Q., Tang, B. Z. (2018) AIE-based super-resolution imaging probes for $\beta$-amyloid plaques in mouse brains. Mater. Chem. Front. 2, 1554-1562. 
[27] Pradhan N., Jana D., Ghorai B.K, and. Jana N.R. (2015) Detection and Monitoring of Amyloid Fibrillation Using a Fluorescence "Switch-On" Probe. ACS Appl. Mater. \& Interfaces. 7 (46), 25813-25820

[28]. Trott, O. \& Olson, A. J. (2010) AutoDock Vina: improving the speed and accuracy of docking with a new scoring function, efficient optimization, and multithreading. J. comput chem. 31 (2), 455-461.

[29]. Morris, G. M. et al. (2009) AutoDock4 and AutoDockTools4: Automated docking with selective receptor flexibility. J. comput chem. 30 (16), 2785-2791.

[30]. Luhrs, T. et al. (2005) 3D structure of Alzheimer's amyloid- (1-42) fibrils. Proc. Natl. Acad. Sci. 102 (48), 17342-17347.

[31] Kuang G, Murugan NA, Tu Y, Nordberg A, Ågren H. (2015) Investigation of the Binding Profiles of AZD2184 and Thioflavin T with Amyloid- $\beta(1-42)$ Fibril by Molecular Docking and Molecular Dynamics Methods. J Phys Chem B. 119 (35), 11560-11567

[32] P. Patel, K. Parmar, D. Patel, S. Kumar, M. Trivedi, M. Das, (2018) Inhibition of amyloid fibril formation of lysozyme by ascorbic acid and a probable mechanism of action. Int. J. Biol. Macromol. 114, 666-678.

[33] Patel, B., Patel, D., Parmar, K., Chauhan, R., Singh, D.D., Pappachan. A. (2018) L. donovani XPRT: Molecular characterization and evaluation of inhibitors. Biochim Biophys Acta Proteins Proteom. 1866 (3), 426-441.

[34] Luhrs, T., Ritter, C., Adrian, M., Riek-Loher, D., Bohrmann, B., Dobeli, H., Schubert, D., Riek, R. (2005) 3D structure of Alzheimer's amyloid-beta(1-42) fibrils. Proc. Natl. Acad. Sci. 102 (48), 17342-17347.

[35] Van Der Spoel, D., Lindahl, E., Hess, B., Groenhof, G., Mark, A.E., Berendsen, H. J. C. (2005) GROMACS: fast, flexible, and free. J. Comput. Chem. 26 (16), 1701-1718. 
[36] Schüttelkopf, A.W., Van Aalten, D.M. (2004) PRODRG: a tool for high-throughput crystallography of protein-ligand complexes. Acta. Crystallogr. D. Biol. Crystallogr. 60 (pt 8), 1355-1363.

[37] Humphrey, W., Dalke, A., Schulten, K., (1996) VMD: Visual molecular dynamics. J. Mol. Graph. 14 (1), 33-38.

[38] Pettersen, E.F., Goddard, T.D., Huang, C.C., Couch, G.S., Greenblatt, D.M., Meng, E.C., Ferrin, T.E. (2004) UCSF Chimera--a visualization system for exploratory research and analysis. J. Comput. Chem. 25 (13), 1605-1612.

[39] Baker, N.A., Sept, D., Joseph, S., Holst, M.J., McCammon, J.A. (2001) Electrostatics of nanosystems: application to microtubules and the ribosome. Proc. Natl. Acad. Sci. 98 (18) 10037-10041.

[40] Kumari, R., Kumar, R., Lynn, A., (2014) g_mmpbsa--a GROMACS tool for highthroughput MM-PBSA calculations. J. Chem. Inf. Model. 54 (7), 1951-1962.

[41]. Grummt, U-W., Weiss, D., Birckner, E., Beckert, R. (2007) Pyridylthiazoles: Highly Luminescent Heterocyclic Compounds. J. Phys. Chem. A. 111 (6), 1104-1110.

[42] Bu F., Duan R., Xie Y., Yi Y., Peng Q., Hu R., Qin A., Zhao Z., Tang BZ. (2015) Unusual Aggregation-Induced Emission of a Coumarin Derivative as a Result of the Restriction of an Intramolecular Twisting Motion. Angew Chem Int Ed Engl. 54 (48), 14492-14497

[43] Ren, Y., Lam, J. W. Y., Dong, Y., Tang, B. Z., Wong, K. S. (2005) Enhanced Emission Efficiency and Excited State Lifetime Due to Restricted Intramolecular Motion in Silole Aggregates. J. Phys. Chem. B. 109 (3), 1135-1140.

[44] Gao, B.R., Wang, H.Y., Hao, Y.W., Fu, L.M., Fang, H.H., Jiang, Y., Wang, L., Chen, Q.D., Xia, H., Pan, L.Y., Ma, Y.G., Sun, H. B. (2010) Time-Resolved Fluorescence Study of Aggregation-Induced Emission Enhancement by Restriction of Intramolecular Charge Transfer State. J. Phys. Chem. B. 114 (1), 128-134. 
[45]. Hong, Y., Lam, J. W. Y., Tang, B. Z. (2011) Aggregation-Induced Emission. Chem. Soc. Rev. 40, 5361-5388.

[46]. Ding, D., Li, K., Liu, B., Tang, B. Z. (2013) Bioprobes Based on AIE Fluorogens. Acc. Chem. Res. 46 (11), 2441-2453.

[47]. Mei, J., Leung, N. L. C., Kwok, R. T. K., Lam, J. W. Y., \& Tang, B. Z. (2015) Aggregation-Induced Emission: Together We Shine, United We Soar! Chem. Rev. 115 (21), 11718-11940

[48] Pustovit, V. N., Shahbazyan, T. V. (2012) Fluorescence quenching near small metal nanoparticles. J. Chem. Phys. 136 (20), 204701.

[49] Faller, P. (2009) Copper and Zinc Binding to Amyloid- $\beta$ : Coordination, Dynamics, Aggregation, Reactivity and Metal- Ion Transfer. Chembiochem. 10 (18), 2837-2845.

[50] Xie, Q., Shi, D., Wan, J., Wang G., et al. (2018) Target regulated photo induced electron transfer of DNA-Cu nanoparticles and their application for the detection of the hepatitis B gene Anal. Methods, 10, 2614-2622.

[51] Rahimi, Y., Goulding, A., Shrestha, S., Mirpuri, S., and Deo S. K. (2008) Mechanism of copper induced fluorescence quenching of red fluorescent protein, DsRed. Biochem Biophys Res Commun. 370 (1), 57-61.

[52] Blatt, E., Chatelier, R. C. and Sawyer, W. H., (1986) Effects of quenching mechanism and type of quencher association on stern-volmer plots in compartmentalized systems. Biophy J. 50 (2), 349-356.

[53] Lakowicz J.R., (2006) Plasmonics in Biology and Plasmon-Controlled Fluorescence Springer, 1 (1), 5-33. 
[54] Murayama K, Tomida M. Heat-induced secondary structure and conformation change of bovine serum albumin investigated by Fourier transform infrared spectroscopy. Biochemistry. 2004

[55]

[56]. Brahmachari, S., Arnon, Z. A., Frydman-Marom, A., Gazit, E. and Adler-Abramovich L. (2017) Diphenylalanine as a Reductionist Model for the Mechanistic Characterization of $\beta$ Amyloid Modulators. ACS Nano. 11 (6), 5960-5969.

[57]. Stine, W. B., Jungbauer, L., Yu, C., Ladu, M. J. (2011) Preparing synthetic A $\beta$ in different aggregation states. Methods.Mol Biol. 670, 13-32.

[85] H. Ren, B. P. Fingerhut, S. Mukamel (2013) Time Resolved Photoelectron Spectroscopy of Thioflavin T Photoisomerization; A Simulation Study. J Phys Chem A. 117(29), 6096-6104.

[58] Groenning M., Norrman M., Flink J.M., van de Weert M., Bukrinsky J.T., Schluckebier G., Frokjaer S.(2007) Binding mode of Thioflavin T in insulin amyloid fibrils. J. Struct. Biol., 159 (3), 483-497.

[59].Biancalana M. and Koide, S. (2010) Molecular mechanism of Thioflavin-T binding to amyloid fibrils. Biochim Biophys Acta. 1804 (7), 1405-1412.

[60]. Groenning, M. (2010) Binding mode of Thioflavin T and other molecular probes in the context of amyloid fibrils-current status, current status. J. Chem. Biol. 3 (1), 1-18.

[61] Wu C., Wang Z., Lei H., Duan Y., Bowers M.T., Shea J.E. (2008) The binding of thioflavin $\mathrm{T}$ and its neutral analog BTA-1 to protofibrils of the Alzheimer's disease Abeta(16-22) peptide probed by molecular dynamics simulations. J. Mol. Biol., 384 (3), 718-729.

[62] Benesi M. L. and Hildebrand, J. H. (1949) A Spectrophotometric Investigation of the Interaction of Iodine with Aromatic Hydrocarbons. J. Am. Chem. Soc., 71 (8), 2703-2707. 
[63] Mora, A. K., Singh P. K., Patro B.S. and Sukhendu Natha, PicoGreen: a better amyloid probe than Thioflavin-T. Chem. Commun., 2016, 52, 12163.

[64] Kuang G., Murugan N.A., Tu Y., Nordberg A., Ågren H., (2015) Investigation of the Binding Profiles of AZD2184 and Thioflavin T with Amyloid- $\beta$ (1-42) Fibril by Molecular Docking and Molecular Dynamics Methods. J Phys Chem B. 119 (35),11560-11567.

\section{Figures and Scheme}
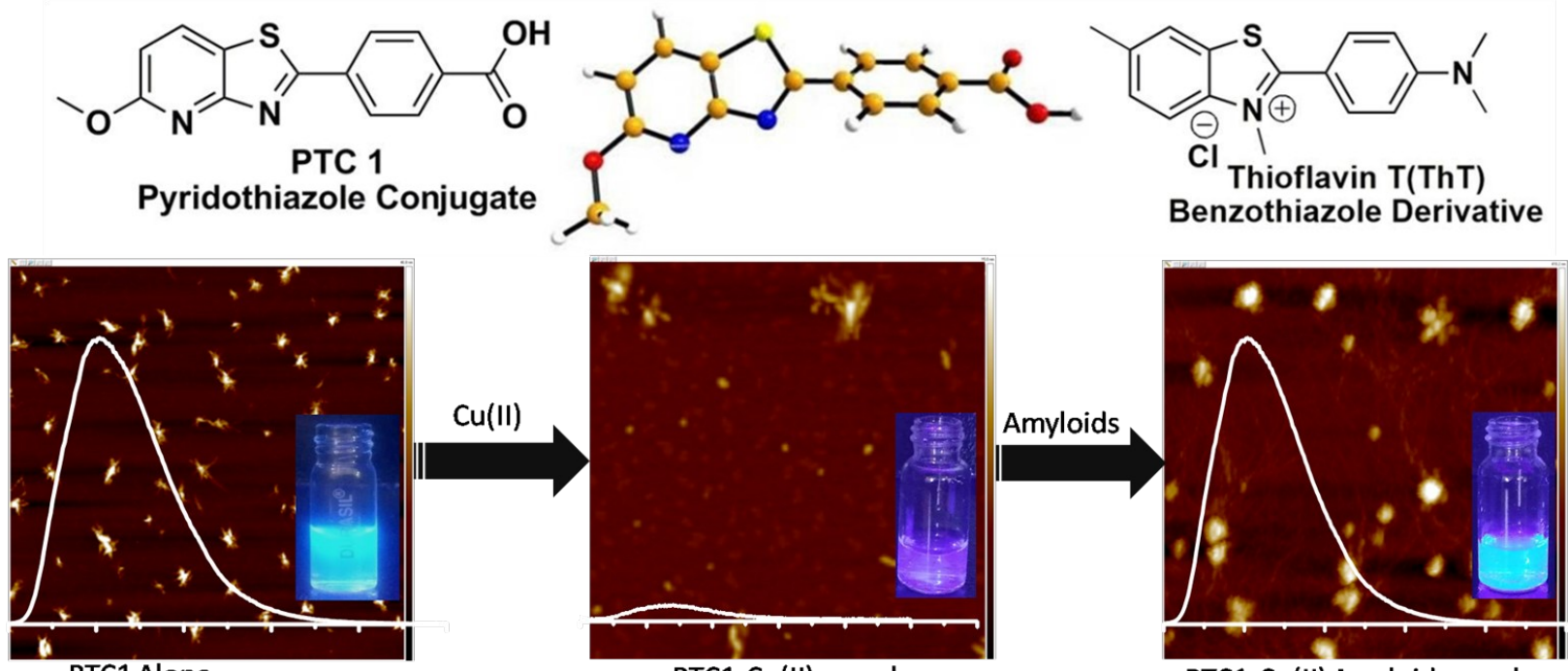

PTC1 Alone

PTC1-Cu(II) complex

PTC1-Cu(II) Amyloid complex

Figure 1. Depicts Top: the basic skeleton of the designed new pyridothiazole based construct

(PTC1) and its energy minimized structure; along with the structure of Thioflavin $\mathrm{T}$ from which

the structure of PTC1 is derived. Bottom: Schematic representation of PTC1 binding assay for detection of amyloid. PTC1 shows good fluorescence which is quenched on the addition of $\mathrm{Cu}^{2+}$ and regenerated on the addition of amyloid. AFM and fluorescence studies reveal that the 
fluorescence enhancement occurs by the aggregation while the quenching occurs by the disaggregation. Representative vial images of PTC1 $(100 \mu \mathrm{M})$ show blue emission under long $\mathrm{UV}$ in the chamber $(365 \mathrm{~nm})$; Fluorescence is quenched by the addition of $\mathrm{Cu}^{2+}$ and regenerated by the addition of amyloid.
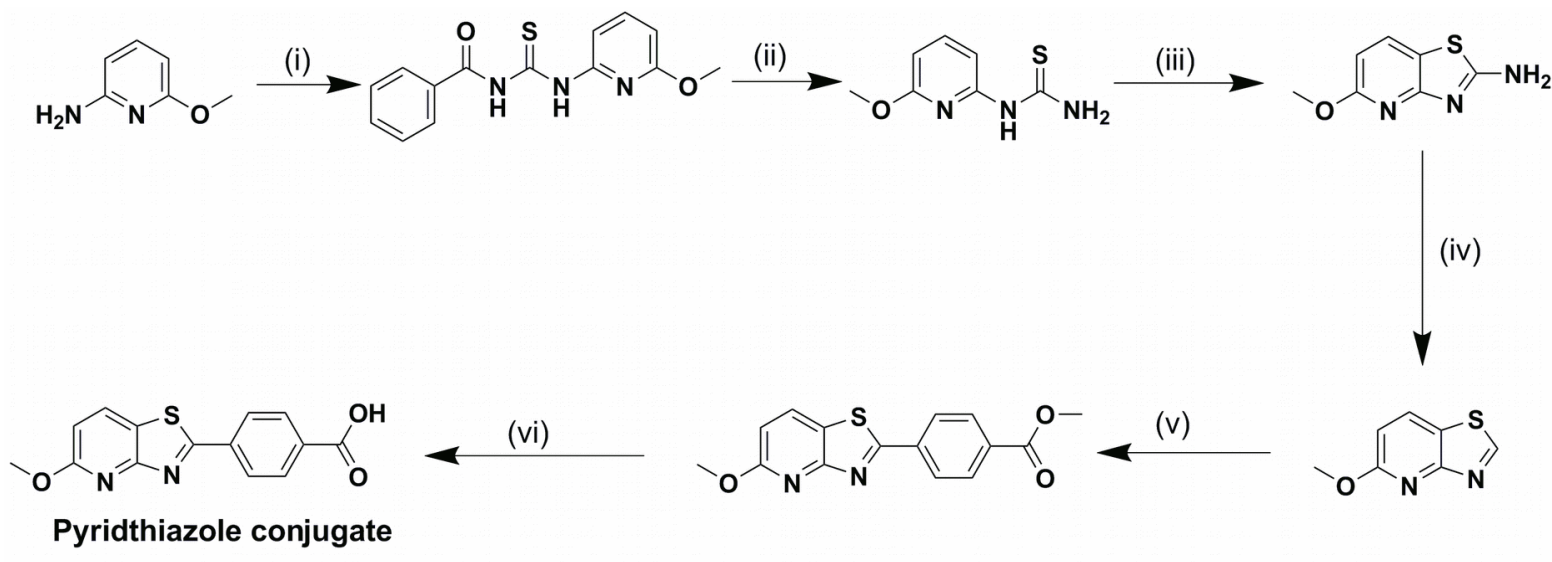

(PTC 1)

Scheme-1: Synthesis of the new molecule PTC1. i) benzoyl isothiacyante, acetone, rt for 2-3 h;

ii) aqueous $\mathrm{NaOH}$, THF, rt, 3h; iii) acetic acid $\mathrm{Br}_{2}, \mathrm{LiBr}, 60{ }^{\circ} \mathrm{C}, 3-4 \mathrm{~h}$; iv) tert-Butyl nitrite, in DMF $70^{\circ} \mathrm{C}$, 3h; v) 4-bromophenyl acetate $\mathrm{Cs}_{2} \mathrm{CO}_{3}$, palladium acetate, $\mathrm{Cu}(\mathrm{I}) \mathrm{Br}$, Xantphos, tolulne, $120{ }^{\circ} \mathrm{C} 16 \mathrm{~h}$; vi) Lithium hydroxide, THF, rt, $4 \mathrm{~h}$. 

Figure 2. (A) Excitation and Emission spectra of PTC1; AFM micrograph of PTC1 $10 \mu \mathrm{M}$. (B)

PTC1 (fresh); (C) PTC1 (5 days); (D) PTC1 twisted fibers (15 days), the inset shows magnified images of PTC1 twisted fibers.
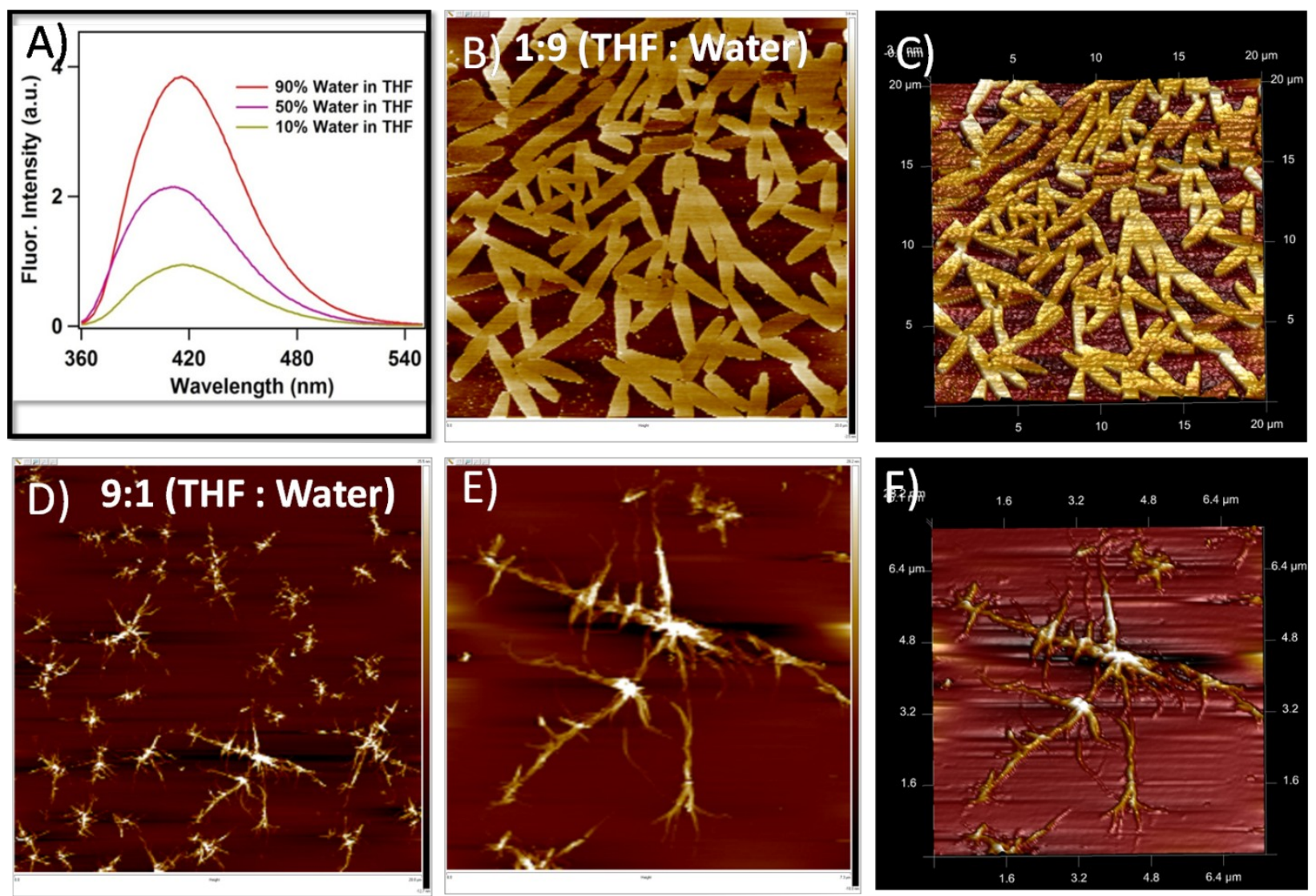

Figure 3: (A) Fluorescence spectra of PTC1 in THF:Water mixture; AFM micrograph of PTC1

$10 \mu \mathrm{M}$ in (B \& C) 2D and corresponding 3D image of PTC1 in 90\% water in THF; (D) 2D AFM image of PTC1 in 10\% water in THF; (E \& F) High resolution 2D and corresponding 3D image of PTC1 in 10\% water in THF. 

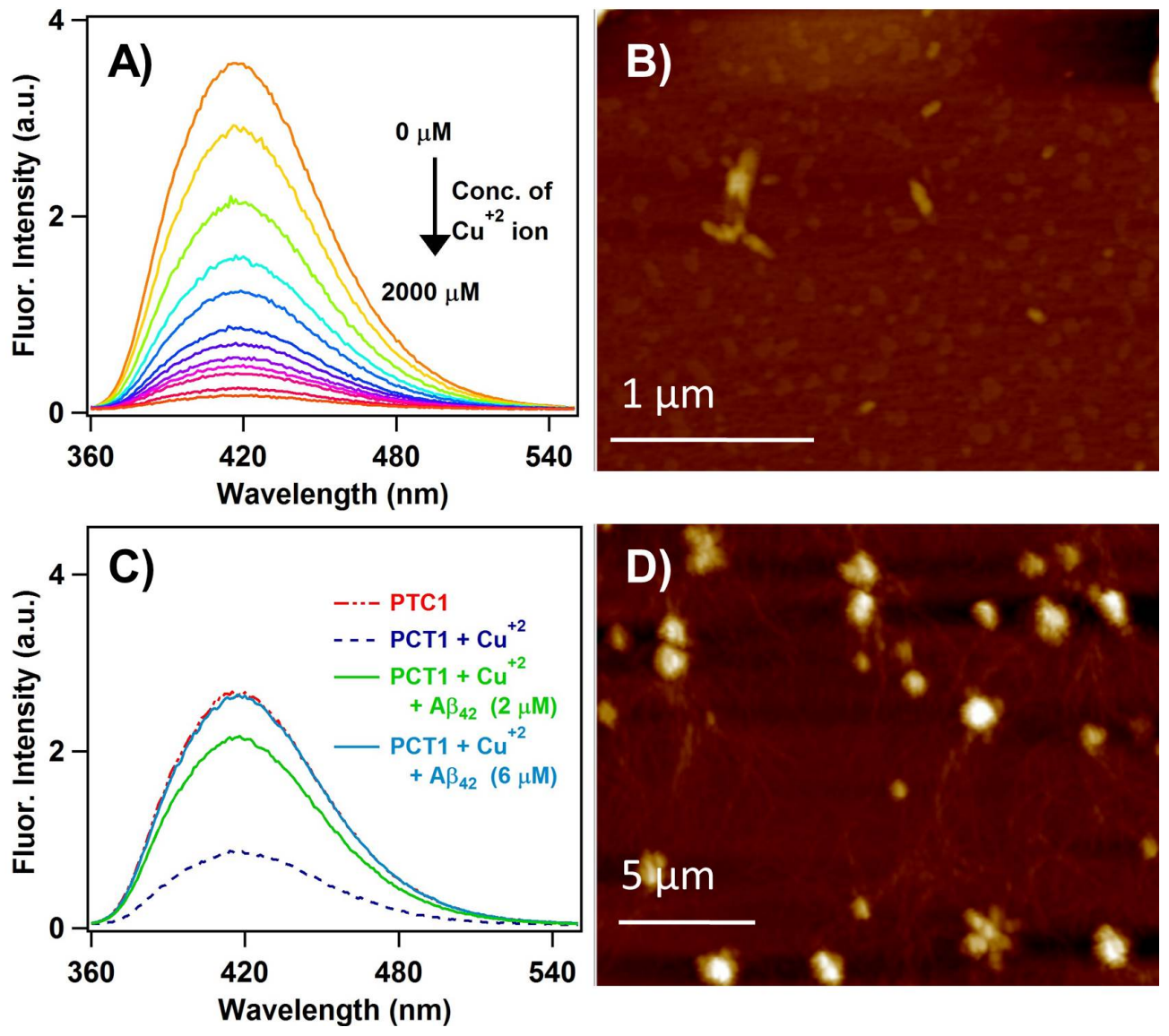

Figure 4: (A) Quenching of PTC1 fluorescence by addition of $\mathrm{Cu}^{2+}$ metal ions and (B) corresponding AFM micrograph showed the disaggregation of PTC1 assembly; (C) Recovery of 
fluorescence in the presence of Amyloidogenic fibers when added to PTC1-Cu(II) complex solution and (D) corresponding AFM micrograph showed binding of PTC1 with amyloids.
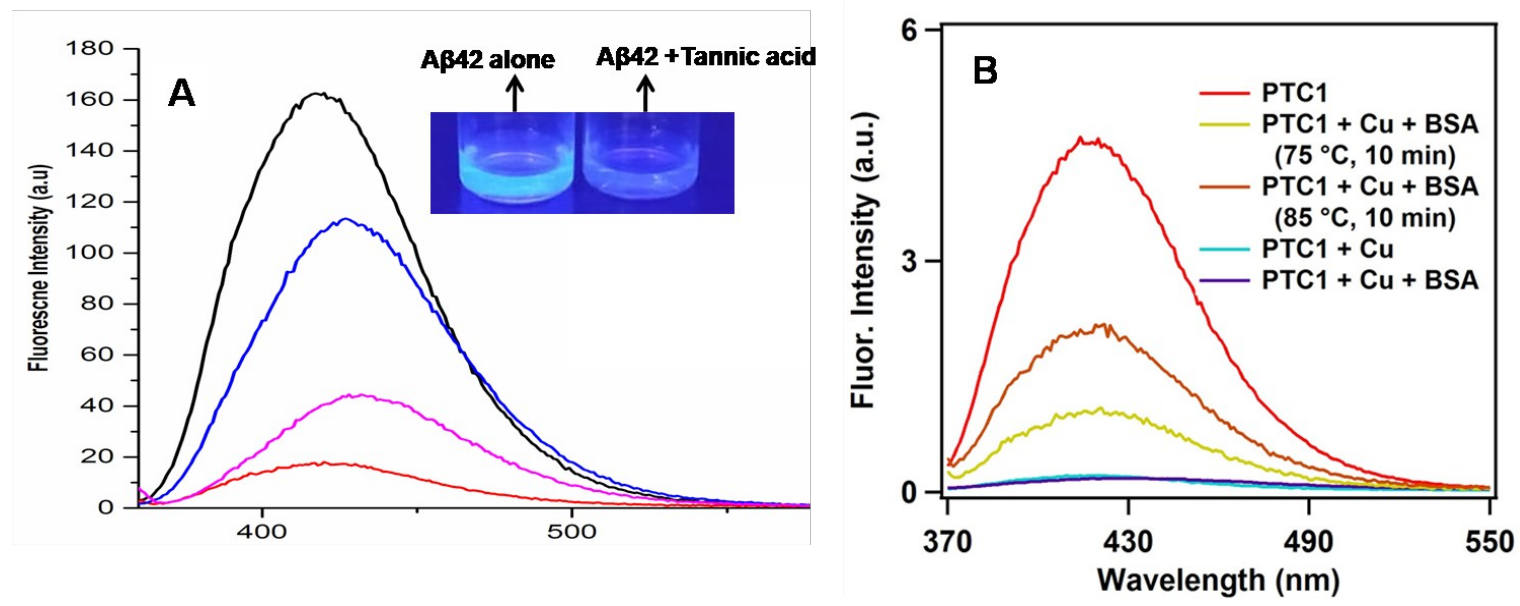

Figure 5: (A) PTC1 assay with FF and FF+Tannic acid (amyloid inhibitor), (inset)shows vials of FF and FF with Tannic acid)0; (B) SEM image of FF and (C) SEM image with FF+amyloid inhibitor Tannic acid. 

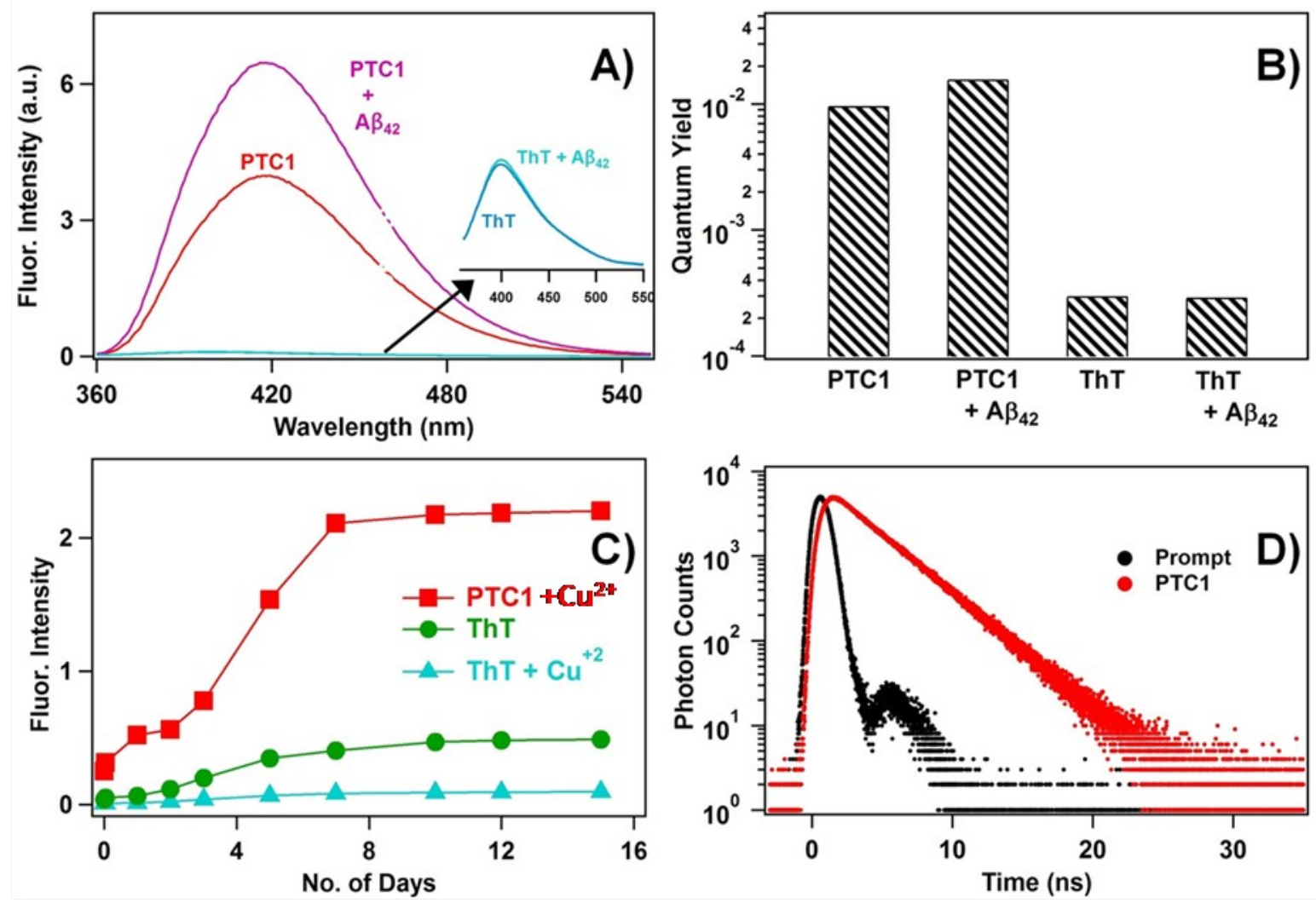

Figure 5: (A) Fluorescence spectra of PTC1 and ThT in presence of 0.5 micromole amyloid;

(B) Quantum yield of PTC1 and ThT in presence and absence of amyloid; (C): A comparative analysis of PTC1 (square) and ThT (triangle) in presence of $\mathrm{Cu}^{2+}(1 \mathrm{mM})$ and 5 micromole $\mathrm{A} \beta_{42}$ aged for 1, 2, 3, 5, 7, 10, 12 and 15 days. ThT (circle) binding assay was also preformed without $\mathrm{Cu}^{2+}$; (D) Fluorescence lifetime of PTC1. 


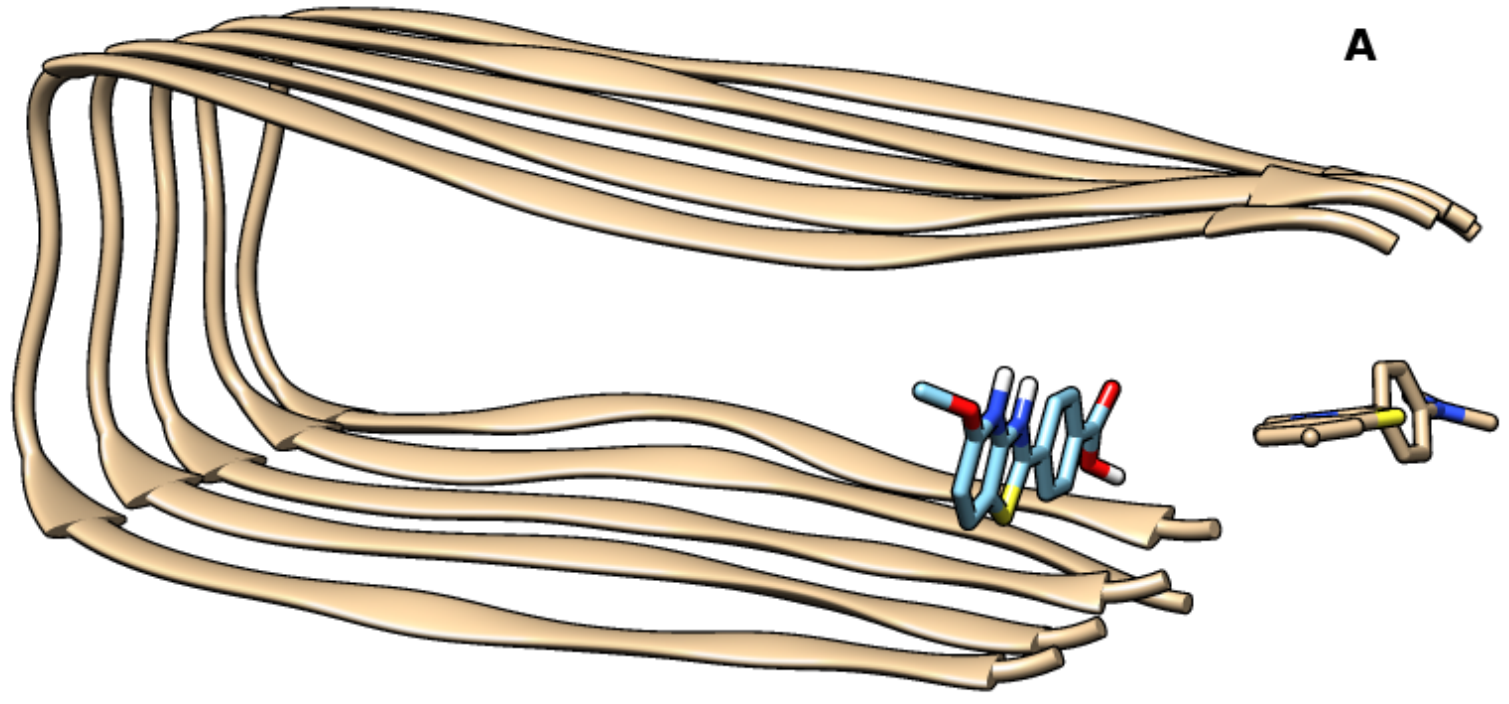

B
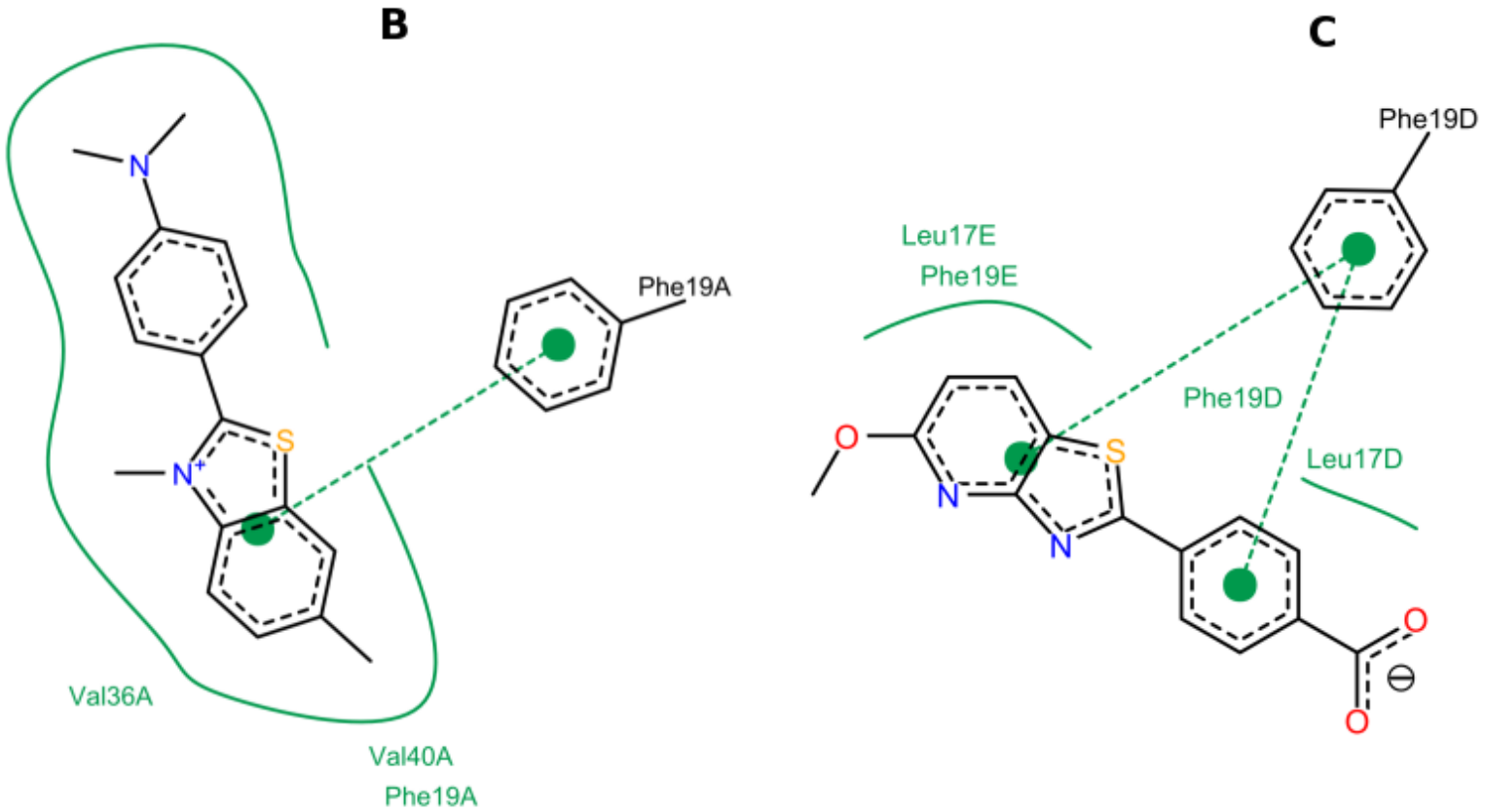

Figure 6: The binding mode of PTC1 and ThT in the entry site of the five $\mathrm{A} \beta_{42}$ fibrils. (A) The $\mathrm{A} \beta_{42}$ fibril is shown in cartoon mode, the binding site residues in stick mode and PTC1 (blue) and ThT (brown) in stick mode; (B) 2D diagram for ThT interaction with $\mathrm{A} \beta_{42}$ residues; (C) 2D diagram for PTC1 interaction with $\mathrm{A} \beta_{42}$ residues. 

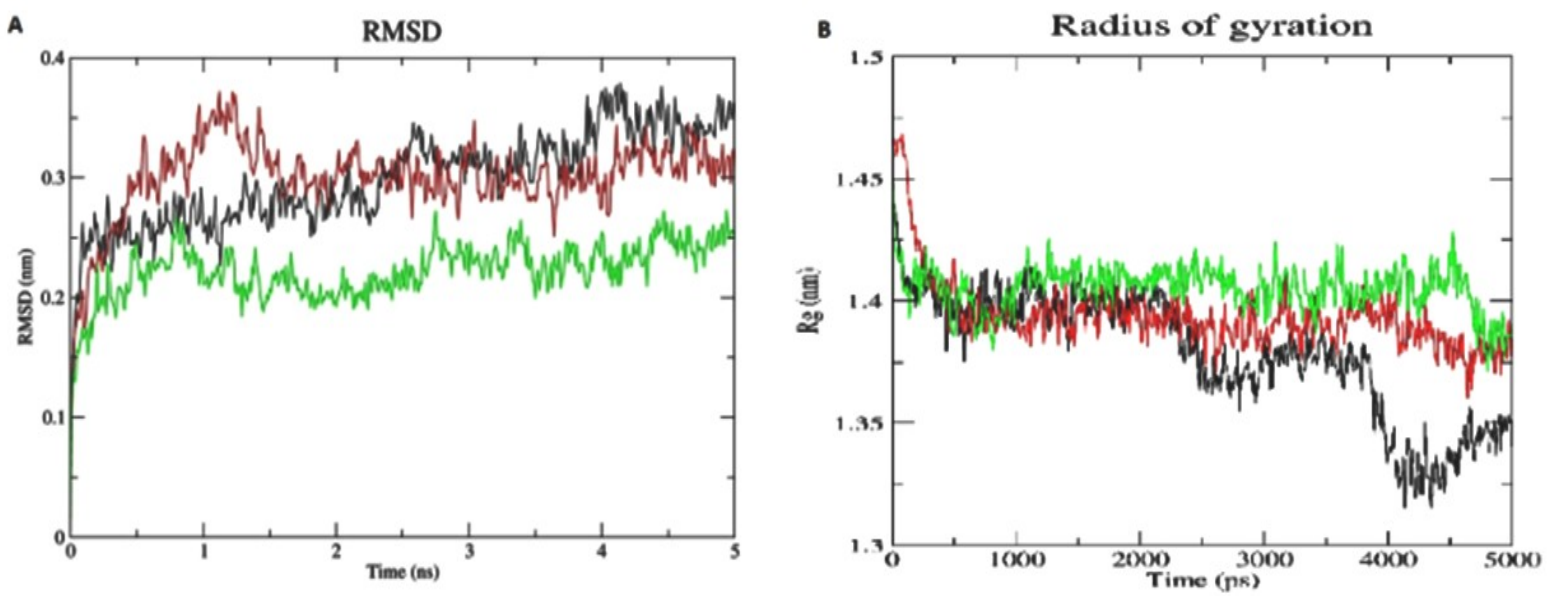

c

D
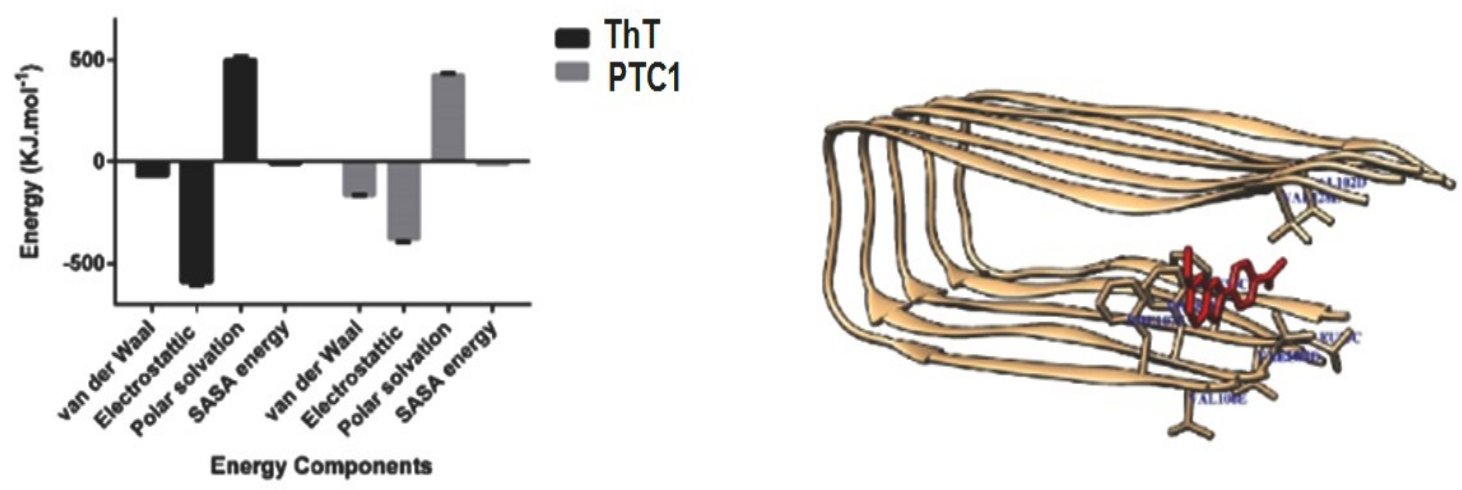

Figure7. Molecular Dynamics analysis of $\mathrm{A} \beta_{42}, \mathrm{~A} \beta_{42}$-ThT, and $\mathrm{A} \beta_{42}$-PTC1. (A) RMSD of the $\mathrm{C}_{\alpha}$ backbone atoms. (B) Computed $\mathrm{R}_{\mathrm{g}}$ of $\mathrm{C} \alpha$ backbone. (C) Representative contributions of each energy component in the total binding free energy for $\mathrm{A} \beta_{42}$-ThT, and $\mathrm{A} \beta_{42}$-PTC1 complexes calculated for entire trajectory at $100 \mathrm{ps}$ interval. (D) MDS snapshot of A $\beta_{42}$-PTC1 complex simulations indicating the molecular interactions labeled with Phe, Val,and Leu residues. 

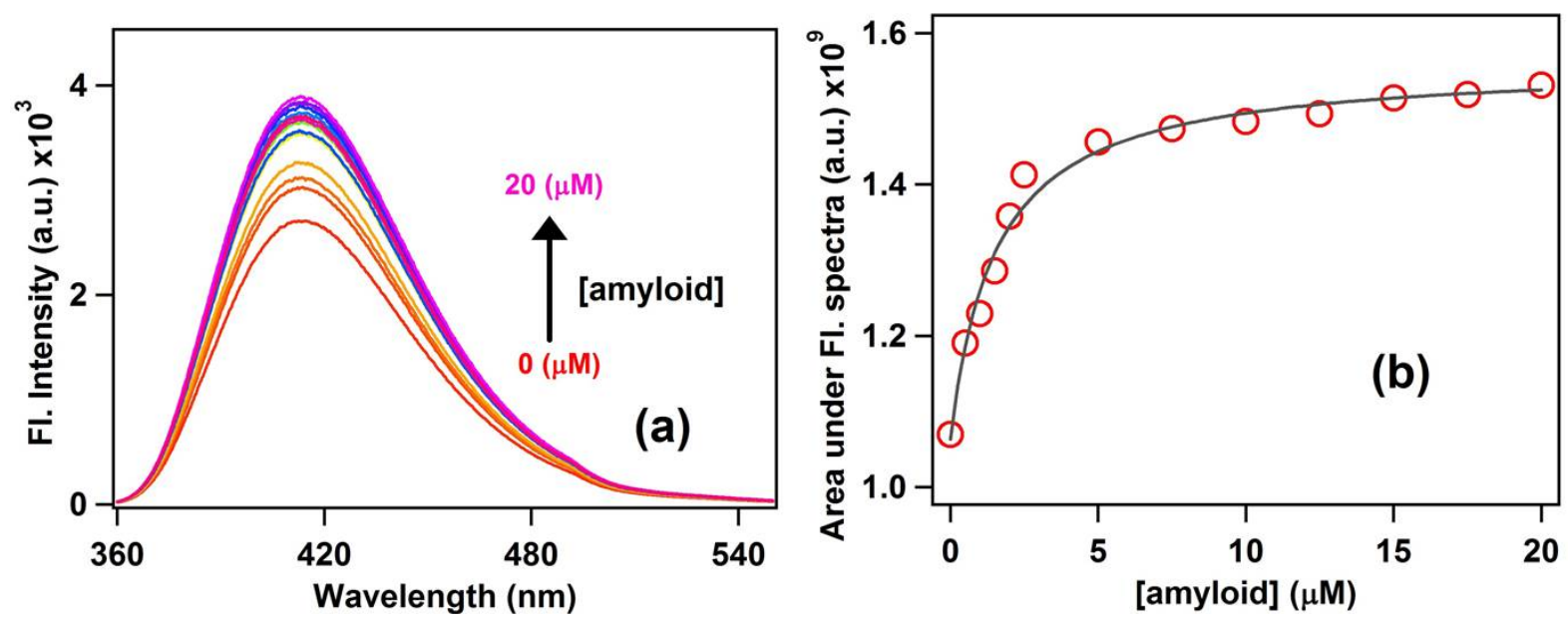

Figure 8. (a) Fluorescence titration of molecule PTC1 in aqueous solution in the presence of different concentrations of 15 days aged $\mathrm{A} \beta 42$ fibrils $(0-20 \mu \mathrm{M})$; (b) The variation of the area under fluorescence spectra with the amyloid concentration and the black solid line represents the best fitting by the equation. 

